\title{
Spinor and Twistor Geometry in Einstein Gravity and Finsler Modifications
}

\author{
Sergiu I. Vacaru
}

\begin{abstract}
We present a generalization of the spinor and twistor geometry for (pseudo) Riemannian manifolds enabled with nonholonomic distributions or for Finsler-Cartan spaces modelled on tangent Lorentz bundles. Nonholonomic (Finsler) twistors are defined as solutions of generalized twistor equations determined by spin connections and frames adapted to nonlinear connection structures. We show that the constructions for local twistors can be globalized using nonholonomic deformations with "auxiliary" metric compatible connections completely determined by the metric structure and/or the Finsler fundamental function. We explain how to perform such an approach in the Einstein gravity theory formulated in Finsler like variables with conventional nonholonomic $2+2$ splitting.
\end{abstract}

Keywords. Spinors and twistors, Finsler geometry, nonlinear connections, nonholonomic manifolds, Einstein spaces.

\section{Introduction}

Twistor theory began with R. Penrose's two papers in 1967 and 1968 and the subject has grown in different directions of modern mathematics and classical and quantum physics (for introductions to twistor theory and reviews of results, and references, see the monographs $[1,2,3,4])$. From a "modest" geometric point of view, twistor structures and transforms are naturally related to certain methods of constructing solutions for self-dual Yang-Mills and Einstein equations and complex/supersymmetric generalizations of the Minkowski and Einstein spacetime geometry.

In this article, we give an introduction into the differential geometry of Finsler spinors and twistors. We shall define twistors for models of metric compatible Finsler spaces and study possible connections to the general relativity theory and modifications. Our "pragmatic" goal is to point researchers that there is an important relation between the twistor transforms, nonholonomic deformations of fundamental spacetime geometric objects and 
a method for generating exact off-diagonal solutions of gravitational field equations in Einstein gravity and modified/generalized theories. ${ }^{1}$ We shall apply a geometric formalism related to the anholonomic frame deformation method of constructing exact solutions [5] and A-brane, deformation and gauge like quantization of gravity [8]. In such models, Finsler like variables can be introduced via respective nonholonomic $2+2$ and/or $n+n$ splitting, for distributions with fibered structure, on Einstein and/or (pseudo) Riemannian manifolds. This allows us to decouple the Einstein equations with respect to some classes of nonholonomic frames and construct generic off-diagonal exact solutions depending on all coordinates via certain classes of generating and integration functions and parameters.

Let us emphasize some substantial differences between the geometry of (pseudo) Finsler spaces and that of (pseudo) Riemannian manifolds ${ }^{2}$, see definitions and details in next section. Different models of Finsler geometry are characterized by different classes of nonlinear and linear connections and lifts on tangent bundles of geometric objects, nonholonomic distributions and related curvature and torsion tensors. Such a geometry is not completely determined by a Finsler metric $F\left(x^{i}, y^{a}\right)$, which defines a nonlinear quadratic element with homogeneity conditions of typical fiber coordinates $y^{a}$ on a tangent bundle $T M$ to a manifold $M$, with local coordinates $x^{i}$, or a (nonholonomic) manifold $\mathbf{V}$ with non-integrable fibred structure. The geometric constructions on Finsler spaces have to be adapted to an another fundamental geometric object, the nonlinear connection (N-connection), $\mathbf{N}$. There are necessary additional assumptions on a chosen Finsler linear connection D (the third fundamental geometric object, also adapted to the $\mathrm{N}$-connection) which can be metric compatible, or non-compatible. ${ }^{3}$ In certain cases, it is possible to construct an associated metric structure $\mathbf{g}$ on total space $T M$, to introduce various types of curvature, torsion and nonmetricity tensors etc.

In brief, a model of Finsler geometry is completely stated by a triple of fundamental "boldface" geometric objects $(F: \mathbf{N}, \mathbf{g}, \mathbf{D})$ generated by $F$,

\footnotetext{
${ }^{1}$ The corresponding metrics can not be diagonalized via coordinate transforms. In modern literature, there are used different words "anholonomic", "nonholonomic" and/or "nonintegrable" which we shall consider as equivalent ones.

${ }^{2}$ In our works, the meaning of the word "pseudo" is equivalent to "semi" which are used for models of curved spaces in standard particle and gravity physics and/or in mathematical literature when the metric may have a local pseudo-Euclidean local signature of type $(-,+,+,+)$. Here we note that such terms, together with a nonstandard for physicists concept of Minkowski space, have different definitions in some monographs on Finsler geometry $[9,10,11,12,13]$, see also recent theories with (pseudo) Finsler metrics, for instance, $[14,15,16,17,18]$.

${ }^{3}$ We use boldface symbols for spaces enabled with a $\mathrm{N}$-connection structure and geometric objects adapted to such a structure [19]. In certain canonical models of Finsler geometry, the values ${ }_{F} \mathbf{N},{ }_{F} \mathbf{g},{ }_{F} \mathbf{D}$ are derived for a fundamental (generating) Finsler function $F$ following certain geometric/variational principles, up to certain classes of generalized frame transforms. For simplicity, in this work we shall omit left up or low labels if that will not result in ambiguities. We shall work on necessary type real and/or complex manifolds of finite dimensions. Tensor and spinor indices will be considered, in general, as abstract ones, and with the Einstein's summation rule for frame/coordinate reprezentations.
} 
which is very different from the case of (pseudo) Riemannian geometry which is determined by data $(\mathbf{g}, \nabla)$, for a metric tensor $\mathbf{g}$ being compatible with the Levi-Civita connection $\nabla$ and with zero torsion (such a linear connection is completely defined by the metric structure).

Some classes of Finsler geometries and generalizations may admit spinor formulations [20, 21, 22]. For instance, in the case of metric compatible models, such methods were developed for the so-called Finsler-Cartan and canonical distinguished connections $[23,14,19])$ when well defined Finsler-Ricci flow [24], supersymmetric [25] and/or noncommutative generalizations [26] can be performed and applied for generating exact solutions for Finsler brane cosmology [27] etc. Such constructions are technically very cumbersome and, in general, not possible for metric noncompatible Finsler geometries with, for instance, Chern or Berwald connections, see [19, 28] and references therein for reviews of results and critical remarks on possible applications in modern physics.

A proposal how the concept of twistors and nonholonomic twistor equations could be extended for Finsler spaces and generalizations was discussed in [21]. The approach was based on an idea to use spinor and twistor geometries for arbitrary finite dimensional spacetimes (see Appendix to volume 2 in [1]) modified to the case of metric compatible Finsler connections and their higher order generalizations with "nonhomogeneous" generating functions, for instance, generalized Lagrange geometries etc. Those nonholonomic (generalized Finsler) twistor constructions had some roots to former our works on "twistor wave function of Universe" [29] and nearly geodesic maps of curved spaces and twistors [30]. ${ }^{4}$

\footnotetext{
${ }^{4}$ Historical Remarks: The author of this paper began his research on "twistor gauge models of gravity" when he was a post-graduate at physics department of "M. A. Lomonosov" State University at Moscow, during 1984-1987. At that time, there were translated in Russian some fundamental papers on Twistor Theory and published a series of important works by "soviet" authors, for instance, the Russian variant of [2]. Various subjective issues related to the "crash" of former Soviet Union resulted in defending author's PhD thesis [31] in 1994, at University Alexandru Ioan Cuza, UAIC, at Iaşi (Yassy), Romania. There were also certain important scientific arguments to transfer such a research on geometry and physics from Russia to Romania, where some schools on nonholonomic geometry and generalized Finsler spaces (for instance, supervised by G. Vrănceanu and A. Bejancu) published a number of works beginning 20ths of previous Century but worked in isolation during dictatorial "socialist period".

Twistor equations are generically nonintegrable for arbitrary curved spacetime. Such equations became integrable, for instance, if the Weyl spinor vanishes, see details in [1]. Our main idea is to use for definition of twistors another class of equations with an "auxiliary" metric compatible connection, completely determined by the same metric but with nontrivial torsion. For certain conditions on noholonomic structure, various "non-integrable" twistor configurations can be globalized in a self-consistent form. Imposing additional anholonomi conditions, we can extract, for instance, certain real vacuum Einstein manifolds. Such methods were formalized by G. Vrănceanu in his geometry of "nonholonomic manifods" [32, 33, 34], see further developments in [35] and, with applications in modern classical and quantum gravity and generalized Finsler geometries and (non) commutative geomeric flows, [14, 19, 26].
} 
Recently, it was suggested [40] to use the nondegenerate Hessian $g_{a b}:=$ $\frac{1}{2} \frac{\partial^{2} F}{\partial y^{a} \partial y^{b}}$ in order to define a class of twistor structures related to Finslerian geodesics, which are integral curves of certain systems of ordinary differential equations (ODEs) and some projective classes of isotropic sprays. Such constructions were performed for Finsler generating functions with scalar flag curvature, in special, for the Randers metric. It was possible to formulate a variational principle for twistor curves arising from such examples of Finsler geometries with scalar flag structure. The (nonlinear) geodesic/ (semi) spray configurations reflect only partially the geometric properties, and possible relations to systems of differential equations (with partial derivatives ones, PDEs, and/or ODEs) of spaces endowed with fundamental Finsler functions. As we emphasized above, complete geometric and possible physically viable models of Finsler spacetimes can be formulated after additional assumptions on linear connection structures and conditions on their (non) compatibility with the metric and $\mathrm{N}$-connection structure. Only in such cases, we can conclude if there are, or not, Finsler analogs of spinors and anisotropic models of bosonic and fermionic fields and interactions for certain classes of Finsler connections.

This paper is organized as follows: In section 2, we present a brief introduction into the geometry of metric compatible nonholonomic manifolds and bundles enabled with nonlinear connection ( $\mathrm{N}$-connection) structure. It is elaborated an unified $\mathrm{N}$-adapted formalism both for the Finsler-Cartan spaces and the Einstein gravity theory reformulated in Finsler like variables. We also provide new results on the geometry of conformal transforms and $\mathrm{N}$-connection structures. Section 3 is devoted to the differential geometry of spinors on Finsler-Cartan and Einstein-Finsler spaces. We define nonholonomic (Finsler) twistors in section 4 considering "auxiliar" metric compatible Finsler like connections. There are studied the conditions when nonholonomic twistors can describe global structures in Einstein gravity and modifications.

In some sense, Finsler spaces are modelled by geometries with nonholonomic distributions on tangent bundles or on manifolds with fibered structure. The most closed to standard physics directions were developed following some fundamental geometric ideas and results due to E. Cartan [23], M. Matsumoto [10] and others (on almost Kähler Finsler structures, Einstein equations for the Cartan-Finsler connection, J. Kern's geometrization of mechanics via Finsler methods [36] etc). Using nonholonomic distributions, it was possible to formulate Clifford and spinor analogs of metric compatible Finsler geometries and generalizations and define Dirac-Finsler operators [20, 21], see also reviews of results and complete lists of references in $[22,14,26]$. Approaches based on Berwald and Chern connections, semisprays and sectional curvature [13, 37] have been developed in modern literature but because of nonmetricity and spectific "nonminimal" relations between the Finsler metric and curvature seem to be less related to standard theories in physics [28, 19]. 


\section{Nonholonomic (Finsler) Geometry and Gravity}

Finsler type geometries can be modelled on (pseudo) Riemannian manifolds and/or tangent bundles enabled with necessary types nonholonomic distributions and supersymmteric and/or noncommutative generalizations. In this section, we fix notations and provide necessary results. For details and proofs, we refer to $[7,19,14]$ where the so-called geometry of Finsler-Einstein gravity and modifications is formulated in a language familiar to researchers in mathematical relativity.

\subsection{The geometry of nonholonomic bundles and manifolds}

2.1.1. Nonlinear and distinguished connections. Let us consider a $(n+m)-$ dimensional real or complex manifold $\mathbf{V}$ of necessary smooth/analytic/holomorphic class, where 1) $n=m=2$ (this will be used for constructing exact solutions in general relativity applying Finsler and twistor methods), or 2) $n=m=4$ (for Finsler twistor models on tangent bundles to Lorentz manifolds).

Definition 2.1. A nonholonomic manifold is a pair $(\mathbf{V}, \mathcal{N})$ defined by a nonintegrable distribution $\mathcal{N}$ on $\mathbf{V}$.

The geometry of nonholonomic real manifolds is studied in Refs. [32, $33,34,19,14]$ for various finite dimensions $n \geq 2$ and $m>1$. For simplicity, we shall consider a subclass of nonholonomic distributions $\mathcal{N}$ stating fibered structures $\pi: \mathbf{V} \rightarrow V$ with constant rank $\pi$, where $V$ is a two dimensional, 2d, or 4-d, (for instance, pseudo-Riemanian manifold, or any its complexified version). In general, we can consider any such map with differential map $\pi^{\boldsymbol{\top}}$ : $T \mathbf{V} \rightarrow T V$ when the kernel of $\pi^{\top}$, which is just the vertical subspace $v \mathbf{V}$ with a related inclusion mapping $i: v \mathbf{V} \rightarrow T \mathbf{V}$, defines a corresponding vertical subspace as a nonholonomic distribution.

Definition and Theorem 2.2. A nonlinear connection ( $N$-connection) $\mathbf{N}$ on $\mathbf{V}$ can be defined in two equivalent forms:

a) by the splitting on the left with an exact sequence $0 \rightarrow v \mathbf{V} \stackrel{i}{\rightarrow} T \mathbf{V} \rightarrow$ $T \mathbf{V} / v \mathbf{V} \rightarrow 0$, i.e. by a morphism of submanifolds $\mathbf{N}: T \mathbf{V} \rightarrow v \mathbf{V}$ such that $\mathbf{N} \circ \mathbf{i}$ is the unity in $v \mathbf{V}$.

b) Globalizing the local distributions associated to such nonholonomic splitting $\mathcal{N}$ we prove that a $N$-connection defines a Whitney sum of conventional horizontal $(h)$ subspace, $h \mathbf{V}$, and vertical $(v)$ subspace, $h \mathbf{V}$,

$$
T \mathbf{V}=h \mathbf{V} \oplus v \mathbf{V} .
$$

We shall use "boldface" symbols in order to emphasize that a geometric object is defined on a nonholonomic manifold $\mathbf{V}$ enabled with $\mathrm{N}$-connection structure and call such an object to be, for instance, a distinguished tensor (in brief, d-tensor, d-metric, d-spinor, $\mathrm{d}$-connection), or a $\mathrm{d}$-vector $\mathbf{X}=$ $(h \mathbf{X}, v \mathbf{X}) \in T \mathbf{V}$. A $\mathrm{N}$-connection is characterized by its curvature, i.e. the Neijenhuis tensor,

$$
\Omega(\mathbf{X}, \mathbf{Y}):=[v \mathbf{X}, v \mathbf{Y}]+v[\mathbf{X}, \mathbf{Y}]-v[v \mathbf{X}, \mathbf{Y}]-v[\mathbf{X}, v \mathbf{Y}],
$$


for any d-vectors $\mathbf{X}, \mathbf{Y}$ and commutator $[\cdot, \cdot] .^{5}$

Definition 2.3. A distinguished connection (d-connection) $\mathbf{D}$ is a linear connection conserving under parallelism the Whitney sum (1), i.e. the $N$-connection splitting into $h$ - and $v$-subspaces.

We can perform a decomposition of $\mathbf{D}$ into h- and v-covariant derivatives $\mathbf{D}=(h \mathbf{D}, v \mathbf{D})$, when $\left.\left.\left.\mathbf{D}_{\mathbf{X}}:=\mathbf{X}\right\rfloor \mathbf{D}=h \mathbf{X}\right\rfloor \mathbf{D}+v \mathbf{X}\right\rfloor \mathbf{D}=h \mathbf{D}_{\mathbf{X}}+v \mathbf{D}_{\mathbf{X}}$, where "」" is the interior product.

Definition 2.4. For a d-connection $\mathbf{D}$, we can define:

a) the torsion d-tensor

$$
\mathcal{T}(\mathbf{X}, \mathbf{Y}):=\mathbf{D}_{\mathbf{X}} \mathbf{Y}-\mathbf{D}_{\mathbf{Y}} \mathbf{X}-[\mathbf{X}, \mathbf{Y}] ;
$$

b) the curvature $d$-tensor

$$
\mathcal{R}(\mathbf{X}, \mathbf{Y}):=\mathbf{D}_{\mathbf{X}} \mathbf{D}_{\mathbf{Y}}-\mathbf{D}_{\mathbf{Y}} \mathbf{D}_{\mathbf{X}}-\mathbf{D}_{[\mathbf{X}, \mathbf{Y}]} .
$$

Introducing $h$ - $v$-decompositions $\mathbf{D}=(h \mathbf{D}, v \mathbf{D})$ and $\mathbf{X}=(h \mathbf{X}, v \mathbf{X})$ in above formulas, we compute respective $h$ - $v$-components (i.e. d-tensor $\mathrm{N}$ adapted components) of the torsion and curvature of a $\mathrm{d}$-connection. For instance, there are five nontrivial components of torsion,

$\mathcal{T}(\mathbf{X}, \mathbf{Y})=\{h \mathcal{T}(h \mathbf{X}, h \mathbf{Y}), h \mathcal{T}(h \mathbf{X}, v \mathbf{Y}), v \mathcal{T}(h \mathbf{X}, h \mathbf{Y}), v \mathcal{T}(a \mathbf{X}, h \mathbf{Y}), v \mathcal{T}(v \mathbf{X}, v \mathbf{Y})\}$ for arbitrary $\mathrm{d}$-vectors $\mathbf{X}$ and $\mathbf{Y}$.

2.1.2. Metric compatible nonholonomic manifolds. A nonholonomic manifold $\mathbf{V}$ is enabled with a metric structure defined by symmetric nondegenerate second rank tensor $\mathbf{g}$. Such a metric can be described equivalently by a $\mathrm{d}-$ metric $\mathbf{g}=(h \mathbf{g}, v \mathbf{g})$ with corresponding $h$ - and $v$-metrics, in $\mathrm{N}$-adapted form.

Definition and Theorem 2.5. Ad-connection $\mathbf{D}$ is metric compatible with a $d-m e t r i c \mathbf{g}$ if and only if $\mathbf{D g}=0$ imposing conditions of compatibility in $h$-v-form for decompositions $\mathbf{D}=(h \mathbf{D}, v \mathbf{D})$ and $\mathbf{g}=(h \mathbf{g}, v \mathbf{g})$.

In this paper, we shall use only metric compatible connections.

Theorem 2.1. Any metric structure $\mathbf{g}$ defines a unique Levi-Civita connection $\nabla$ which is metric compatible, $\nabla \mathbf{g}=0$, and with zero torsion,

$$
\nabla_{\mathcal{T}}(\mathbf{X}, \mathbf{Y}):=\nabla_{\mathbf{X}} \mathbf{Y}-\nabla_{\mathbf{Y}} \mathbf{X}-[\mathbf{X}, \mathbf{Y}]=0
$$

We note that $\nabla$ is not a $\mathrm{d}$-connection because it does not preserve under parallelism the $\mathrm{N}$-connection splitting. For a $\mathbf{N}(1)$ with nonzero $\mathbf{N}-$ curvature $\Omega(2)$, there is a "preferred" $\mathrm{d}$-connection which can be considered as the analog of the Levi-Civita connection for nonholonomic manifolds:

\footnotetext{
${ }^{5} \mathrm{~N}$-connections were used in coefficient form in E. Cartan's first monograph on Finsler geometry [23]. The first global definition is due to C. Ehresmann [38], it was studied in Finsler geometry and generalizations by A. Kawaguchi [39] and Greek and Romanian geometers and physicists [14]. The abroach was developed and applied to various nonholonomic and/or Finsler generalizations on superspaces, in noncommutative geometry and constructing exact solutions in gravity $[20,25,21,14,19]$, see also references therein.
} 
Theorem 2.2. Any metric structure $\mathbf{g}$ defines a unique canonical d-connection $\mathbf{D}$ which is metric compatible, $\mathbf{D g}=0$, and with zero "pure" horizontal and "vertical" d-torsions, i.e., respectively, $h \mathcal{T}(h \mathbf{X}, h \mathbf{Y})=0$ and $v \mathcal{T}(v \mathbf{X}, v \mathbf{Y})$, for $\mathcal{T}(\mathbf{X}, \mathbf{Y}):=\mathbf{D}_{\mathbf{X}} \mathbf{Y}-\mathbf{D}_{\mathbf{Y}} \mathbf{X}-[\mathbf{X}, \mathbf{Y}]$.

For a fixed $\mathbf{N}$, both linear connections $\nabla$ and $\mathbf{D}$ are completely defined by the same metric $\mathbf{g} .{ }^{6}$ There is a substantial difference between the canonical d-connection $\mathbf{D}$ and the connections used in Riemann-Cartan geometry (see, for instance, [1] and detailed discussions with respect Einstein and Finsler geometries and metric-affine generalizations in $[7,19,14])$. The torsion $\mathcal{T}$ is completely defined by data $(\mathbf{g}, \mathbf{N})$, i.e. by the metric structure if the value $\mathbf{N}$ is prescribed, but in the Einstein-Cartan gravity we need additional algebraic equations for torsion.

Corollary 2.1. There is a canonical distortion relation

$$
\mathbf{D}=\nabla+\mathbf{Q},
$$

where both linear connections $\mathbf{D}$ and $\nabla$ and the distortion tensor $\mathbf{Q}$ are completely defined by the metric tensor $\mathbf{g}$ for a prescribed $N$-connection splitting.

Using the torsion tensor $\mathcal{R}(4)$ of $\mathbf{D}$, we can introduce in standard form the Ricci d-tensor Ric, which is nonsymmetric because of nontrivial nonholonomic/ torsion structure, the curvature scalar ${ }_{s} R$ and the Einstein d-tensor $\mathbf{E}$. The (canonical nonholonomic) Einstein equations for $\mathbf{D}$ are written geometrically

$$
\mathbf{E}=\mathbf{R i c}-\frac{1}{2} \mathbf{g}{ }_{s} R=\Upsilon,
$$

where the source $\Upsilon$ can be computed as in general relativity (GR) with spacetime metric but $\nabla \rightarrow$ D. A N-adapted variational calculus with possible matter fields (fluids, bosons, fermions etc, all with respect to nonholonomic frames) can be formulated but this will result in nonsymmetric d-tensors $\Upsilon$, which is not surprising because of nonholonmic character of such constructions with induced torsion. On (pseudo) Riemannian manifolds with singnature $(-+++)$ and a prescribed nonintegrable decomposition $2+2$, we can perform local constructions with $\Upsilon \rightarrow\left\{T_{\alpha \beta}\right\}$, where $T_{\alpha \beta}$ is the energymomentum tensor in GR. This allows us to provide a physical interpretation to interactions constants. We need additional assumptions on new interaction constants if the equations are considered on a tangent bundle with total dimension 8; from a formal geometric point of view, there are certain canonical lifts of geometric objects on Lorentz manifolds to their tangent bundles.

The canonical distortion tensor $\mathbf{Q}[\mathbf{g}, \mathbf{N}]$ from (5) is an algebraic combination of the nonholonomically induced torsion $\mathcal{T}[\mathbf{g}, \mathbf{N}]$ all completely

\footnotetext{
${ }^{6}$ In a series of our works, we wrote $\widehat{\mathbf{D}}$ for the canonical d-connection and used "hats" for the coefficients and values computed for this linear connection. In this article we shall use only the symbol $\mathbf{D}$; in next sections, $\widehat{\mathbf{D}}$ will be used for a conformal transforation of $\mathbf{D}$.
} 
defined by values $\mathbf{g}$ and $\mathbf{N}$. It is possible to choose certain integrable configurations ${ }^{i n t} \mathbf{N}$ (this is equivalent to transforms of geometric constructions with respect to certain classes of locally integrable frames of reference) when

$$
\Omega=0, \mathcal{T}=0, \mathbf{Q}=0
$$

Theorem 2.3. For integrable $N$-connection structures when $\mathbf{D}_{\mid \mathcal{T}=0}=\nabla$, the canonical nonholonomic Einstein equations (6) for (pseudo) Riemannian metrics of dimension $2+2$ are equivalent to the Einstein equations in GR.

Proof. It is a straightforward consequence of Corollary 2.1 and (7) and above presented considerations on sources.

2.1.3. Formulas in $\mathbf{N}$-adapted frames and coordinates. We shall denote the local coordinates on a nonholonomic manifold $\mathbf{V}$ in the form $u=(x, y)$, or $u^{\alpha}=\left(x^{i}, y^{a}\right)$, where the $\mathrm{h}$-indices run values $i, j, \ldots=1,2, \ldots n$ (for nonholonomic deformations in GR, $i, j, \ldots=1,2$ or, on tangent to Lorentz bundles, $i, j, \ldots=1,2,3,4)$ and the $\mathrm{v}$-indices take values $a, b, c, \ldots=n+1, n+2, n+m$ (for nonholonomic deformations in GR, $a, b, \ldots=3,4$ and, on tangent to Lorentz bundles, or $a, b, \ldots=5,6,7,8)$. For bundle spaces, $y^{a}$ are typical fiber coordinates and $x^{i}$ are coordinates on base manifolds. We can introduce on $\mathbf{V}$ certain local coordinate bases $\partial_{\underline{\alpha}}=\partial / \partial u^{\underline{\alpha}}=\left(\partial_{i}=\partial / \partial x^{i}, \partial_{a}=\partial / \partial y^{a}\right)$ and their duals $d u \underline{\underline{\beta}}=\left(d x^{j}, d y^{b}\right)$ [we shall emphasize some indices if it is necessary that they are coordinate ones but omit "underlining" when that will not result in ambiguities].

Transforms to arbitrary local frames, $e_{\alpha}$, and (co) frames, $e^{\beta}$, are given by nondegenerate "vierbein" matrices, $e^{\underline{\alpha}} \alpha(u)$, and their duals, $e^{\beta}{ }_{\underline{\beta}}(u)$, respectively, $e_{\alpha}=e^{\underline{\alpha}} \partial_{\underline{\alpha}}$ and $e^{\beta}=e^{\beta}{ }_{\beta} d u^{\underline{\beta}}$. Such transforms do not preserve a $\mathrm{N}$ connection splitting and mix $h-\bar{v}$-indices. "Not-underlined" indices $\alpha, \beta, \ldots$; $i, j, \ldots ; a, b, \ldots$ will be considered, in general, as abstract labels [1]. The indices may be considered as coordinate ones for decompositions with respect to coordinate bases (in our works, we do not consider "boldface" indices but only "boldface" symbols for spaces/geometric objects enabled with/adapted to a $\mathrm{N}$-connection structure).

Locally, a $\mathrm{N}$-connection $\mathbf{N}(1)$ is defined by its coefficients $N_{i}^{a}(u)$,

$$
\mathbf{N}=N_{i}^{a}(u) d x^{i} \otimes \partial / \partial y^{a} .
$$

Proposition 2.1. A $N$-connection structure states a $N$-linear system of reference,

and its dual

$$
\mathbf{e}_{\alpha}=\left(\mathbf{e}_{i}=\frac{\partial}{\partial x^{i}}-N_{i}^{b} \frac{\partial}{\partial y^{b}}, e_{a}=\frac{\partial}{\partial y^{a}}\right)
$$

$$
\mathbf{e}^{\beta}=\left(e^{j}=d x^{j}, \mathbf{e}^{b}=d y^{b}+N_{i}^{b} d x^{i}\right) .
$$

Proof. This follows from the possibility to construct $\mathrm{N}$-adapted bases of type

$$
\mathbf{e}_{\alpha}=\mathbf{e}_{\alpha} \underline{\alpha} \partial_{\underline{\alpha}} \text { and } \mathbf{e}^{\beta}=\mathbf{e}_{\underline{\beta}}^{\beta} d u \underline{\beta},
$$


where

$$
\mathbf{e}_{\alpha}^{\underline{\alpha}}(u)=\left[\begin{array}{cc}
e_{i}^{\underline{i}}(u) & N_{i}^{b}(u) e_{b} \underline{a}(u) \\
0 & e_{a}^{\underline{a}}(u)
\end{array}\right], \mathbf{e}_{\underline{\beta}}^{\beta}(u)=\left[\begin{array}{cc}
e^{i}{ }_{\underline{i}}(u) & -N_{k}^{b}(u) e^{k}{ }_{\underline{i}}(u) \\
0 & e_{\underline{a}}^{a}(u)^{\underline{a}}
\end{array}\right] .
$$

One of the arguments to say that manifolds/bundles enabled with $\mathrm{N}$ connection structure are nonholonomic is that the frames (9) satisfy the nonholonomy relations

$$
\left[\mathbf{e}_{\alpha}, \mathbf{e}_{\beta}\right]=\mathbf{e}_{\alpha} \mathbf{e}_{\beta}-\mathbf{e}_{\beta} \mathbf{e}_{\alpha}=W_{\alpha \beta}^{\gamma} \mathbf{e}_{\gamma},
$$

where the (antisymmetric) nontrivial anholonomy coefficients are computed $W_{i a}^{b}=\partial_{a} N_{i}^{b}$ and $W_{j i}^{a}=\Omega_{i j}^{a}$.

Proposition 2.2. Any metric structure $\mathbf{g}$ on $\mathbf{V}$ can be written in $N$-adapted form as a distinguished metric (d-metric)

$$
\mathbf{g}={ }^{h} g+{ }^{v} h=g_{i j}(u) e^{i} \otimes e^{j}+h_{a b}(u) \mathbf{e}^{a} \otimes \mathbf{e}^{b} .
$$

Proof. Via frame/coordinate transforms, $g_{\alpha \beta}=e_{\alpha}^{\alpha^{\prime}} e_{\beta}^{\beta^{\prime}} g_{\alpha^{\prime} \beta^{\prime}}$, any metric.

$$
\mathbf{g}=\underline{g}_{\alpha \beta}(u) d u^{\alpha} \otimes d u^{\beta}
$$

can written in the form

$$
\underline{g}_{\alpha \beta}=\left[\begin{array}{cc}
g_{i j}+N_{i}^{a} N_{j}^{b} h_{a b} & N_{j}^{e} h_{a e} \\
N_{i}^{e} h_{b e} & h_{a b}
\end{array}\right] .
$$

Introducing formulas (9) and (11) into (13) we obtain the coordinate form (14) and (15). Inverse transforms are similar.

Using the last two propositions, we can compute the $\mathrm{N}$-adapted coefficients $\Gamma_{\alpha \beta}^{\gamma}=\left(L_{j k}^{i}, L_{b k}^{a}, C_{j c}^{i}, C_{b c}^{a}\right)$, with respect to frames (8) and (9), of the canonical d-connection $\mathbf{D}$,

$$
\begin{aligned}
L_{j k}^{i} & =\frac{1}{2} g^{i r}\left(\mathbf{e}_{k} g_{j r}+\mathbf{e}_{j} g_{k r}-\mathbf{e}_{r} g_{j k}\right), \\
L_{b k}^{a} & =e_{b}\left(N_{k}^{a}\right)+\frac{1}{2} h^{a c}\left(e_{k} h_{b c}-h_{d c} e_{b} N_{k}^{d}-h_{d b} e_{c} N_{k}^{d}\right), \\
C_{j c}^{i} & =\frac{1}{2} g^{i k} e_{c} g_{j k}, C_{b c}^{a}=\frac{1}{2} h^{a d}\left(e_{c} h_{b d}+e_{c} h_{c d}-e_{d} h_{b c}\right) .
\end{aligned}
$$

The N-adapted coefficients of d-torsion

$$
\begin{aligned}
\mathcal{T}=\left\{\mathbf{T}_{\alpha \beta}^{\gamma}\right. & \left.=\left(T^{i}{ }_{j k}, T^{i}{ }_{j a}, T_{j i}^{a}, T_{b i}^{a}, T_{b c}^{a}\right)\right\}(2.1 .1) \text { of } \mathbf{D} \text { are computed } \\
T^{i}{ }_{j k} & =L^{i}{ }_{j k}-L^{i}{ }_{k j}=0, \quad T^{i}{ }_{j a}=-T^{i}{ }_{a j}=C^{i}{ }_{j a}, T_{j i}^{a}=\Omega^{a}{ }_{j i}, \\
T^{a}{ }_{b i} & =\partial_{b} N_{i}^{a}-L^{a}{ }_{b i}, T_{b c}^{a}=C_{b c}^{a}-C^{a}{ }_{c b}=0 .
\end{aligned}
$$

We provide also the $\mathrm{N}$-adapted coefficients of $\mathrm{d}$-curvature, 


$$
\begin{aligned}
& \mathcal{R}=\left\{\mathbf{R}_{\alpha \beta \nu}^{\tau}=\left(R_{h j k}^{i}, R_{b j k}^{a}, R_{j k a}^{i}, R_{b k a}^{c}, R_{j b c}^{i}, R_{b c d}^{a}\right)\right\} \text { (4) of D, } \\
& R_{h j k}^{i}=\mathbf{e}_{k} L_{h j}^{i}-\mathbf{e}_{j} L_{h k}^{i}+L_{h j}^{m} L_{m k}^{i}-L_{h k}^{m} L_{m j}^{i}-C_{h a}^{i} \Omega^{a}{ }_{k j} \text {, } \\
& R_{b j k}^{a}=\mathbf{e}_{k} L_{b j}^{a}-\mathbf{e}_{j} L_{b k}^{a}+L_{b j}^{c} L_{c k}^{a}-L_{b k}^{c} L^{a}{ }_{c j}-C_{b c}^{a} \Omega^{c}{ }_{k j} \text {, } \\
& R^{i}{ }_{j k a}=e_{a} L^{i}{ }_{j k}-D_{k} C^{i}{ }_{j a}+C^{i}{ }_{j b} T^{b}{ }_{k a} \text {, } \\
& R_{b k a}^{c}=e_{a} L_{b k}^{c}-D_{k} C_{b a}^{c}+C_{b d}^{c} T_{k a}^{c} \text {, } \\
& R^{i}{ }_{j b c}=e_{c} C^{i}{ }_{j b}-e_{b} C^{i}{ }_{j c}+C^{h}{ }_{j b} C^{i}{ }_{h c}-C^{h}{ }_{j c} C^{i}{ }_{h b}, \\
& R_{b c d}^{a}=e_{d} C_{b c}^{a}-e_{c} C_{b d}^{a}+C_{b c}^{e} C_{e d}^{a}-C_{b d}^{e} C_{e c}^{a} \text {. }
\end{aligned}
$$

Contracting indices, we can compute the h- $\mathrm{v}$-components $\mathbf{R}_{\alpha \beta} \doteqdot \mathbf{R}^{\tau}{ }_{\alpha \beta \tau}$ of the Ricci tensor Ric,

$$
R_{i j} \doteqdot R_{i j k}^{k}, R_{i a} \doteqdot-R_{i k a}^{k}, R_{a i} \doteqdot R_{a i b}^{b}, R_{a b} \doteqdot R_{a b c}^{c}
$$

The scalar curvature is

$$
{ }_{s} R \doteqdot \mathbf{g}^{\alpha \beta} \mathbf{R}_{\alpha \beta}=g^{i j} R_{i j}+h^{a b} R_{a b} .
$$

In component form, the analog of Theorem 2.3 is

Theorem 2.4. The Einstein equations in GR are equivalent to

$$
\begin{aligned}
\mathbf{R}_{\beta \delta}-\frac{1}{2} \mathbf{g}_{\beta \delta}{ }_{s} R & =\mathbf{\Upsilon}_{\beta \delta}, \\
L_{a j}^{c}=e_{a}\left(N_{j}^{c}\right), C_{j b}^{i}=0, \Omega^{a}{ }_{j i} & =0,
\end{aligned}
$$

written for the canonical d-connection coefficients (16) if $\boldsymbol{\Upsilon}_{\beta \delta} \rightarrow T_{\beta \delta}$ (energy-momentum tensor for matter) for $\mathbf{D} \rightarrow \nabla$.

Proof. It follows from above component formulas introduced in (6) and (7). The constraints (22) are equivalent to (7), i.e. to the condition of zero torsion (17) and zero distortion d-tensors $\mathbf{Q}=0$, which results in $\mathbf{D}=\nabla$, see (5).

The main reason to work with equations of type (6) and (21) is that such equations for D (we say "in nonholonomic variables") decouple with respect to $\mathrm{N}$-adapted frames (for spaces with splitting of dimension 2, or $3,+2+2+2+\ldots)$ for generic off-diagonal ansatz for metric $\mathbf{g}$ and certain parameterizations of $\mathbf{N}$ depending on all coordinates. This allows us to integrate such nonlinear PDE in very general forms. We construct integral varieties determined by corresponding classes of generating and integration functions and integration constant which may be defined from certain boundary/Cauchy conditions and additional physical arguments. Imposing additional Levi-Civita (LC) conditions (7), which constrain nonholonomically the integral varieties of solutions of $\mathbf{E}=\Upsilon$, we can "extract" solutions in GR. We note that we can not decouple and integrate in such off-diagonal forms the Einstein equations if we work from the very beginning and only with $\nabla$. The main "trick" is that we "relax" the constraints of zero torsion in the standard Einstein equations by considering an "auxiliary" connection D (in next section, we shall see that this is a Finsler type d-connection); such constructions are provided in Refs. [5, 14, 19]. 


\subsection{Metric compatible Finsler-Cartan geometries}

We outline some results from the Finsler geometry on tangent bundles [11, 23, $9,10,13]$ and show how the constructions can be re-defined for nonholonomic (pseudo) Riemannian manifolds [19, 14, 28].

2.2.1. The Finsler fundamental/generating function. Let us consider a tangent bundle $T M=\bigcup_{x \in M} T_{x} M$, where $T_{x} M$ are the tangent spaces at points $x \in M$, for the base space $M$ being a real $C^{\infty}$ manifold of dimension $\operatorname{dim} M=n$. Roughly, the term Finsler "metric" $F$ is used for a (Finsler) geometry determined on $T M$ by a nonlinear quadratic element

$$
d s^{2}=F^{2}(x, d x),
$$

when $d x^{i} \sim y^{i}$. This generalizes the well-known and very important example of (pseudo) Riemannian geometry, determined by a metric tensor $g_{i j}\left(x^{k}\right)$. Taking a particular case with quadratic form $F=\sqrt{\left|g_{i j}(x) y^{i} y^{j}\right|}$ we obtain

$$
d s^{2}=g_{i j}(x) d x^{i} d x^{j} .
$$

Such an element states a geometry on $M$ with geometric objects depending only on $x$-variables even for definitions of tensors, linear connections, spinors etc objects the tangent bundle $T M$ is also involved in order to define such objects by analogy to flat spaces.

Definition 2.6. A Finsler fundamental/generating function (metric) is a function $F: T M \rightarrow[0, \infty)$ subjected to the conditions:

1. $F(x, y)$ is $C^{\infty}$ on $\widetilde{T M}:=T M \backslash\{0\}$, for $\{0\}$ denoting the set of zero sections of $T M$ on $M$;

2. $F(x, \beta y)=\beta F(x, y)$, for any $\beta>0$, i.e. it is a positive 1-homogeneous function on the fibers of $T M$;

3. for any $y \in \widetilde{T_{x} M}$, the Hessian

$$
{ }^{v} \tilde{g}_{i j}(x, y)=\frac{1}{2} \frac{\partial^{2} F^{2}}{\partial y^{i} \partial y^{j}}
$$

is considered as s a "vertical" $(v)$ metric on typical fiber, i.e. it is nondegenerate and positive definite, $\operatorname{det}\left|{ }^{v} \tilde{g}_{i j}\right| \neq 0$.

If the base $M$ is taken to be a Lorentz manifold in GR, we can construct generalizations on $T M$ with a good physical axiomatic system which is very similar to that of Einstein gravity when the Levi-Civita connection $\nabla$ is substituted by a metric compatible Finsler variant of the canonical $d$-connection D, see discussions in [41] and next subsections.

Remark 2.1. The condition 3 above should be relaxed to "not positive definite" for models of Finsler gravity with finite, in general, locally anisotropic speed of light.

Considering a background (pseudo) Riemannian metric $g_{i j}(x)$ with signature $(+,+,+,-)$ on $M$, we can elaborate various geometric and physical 
models on $T M$ with locally anisotropic metrics $g_{i j}(x, y)$ depending on "velocity" type coordinates $y^{a}$. The main difference between (pseudo) Riemannian and Finsler geometries is that the first type ones are completely defined by a metric structure $g_{i j}(x)$ (from which a unique Levi-Civita connection $\nabla$ can be constructed) but the second type ones can not be completely derived from a Finsler metric $F(x, y)$ and/or its Hessian ${ }^{v} \tilde{g}_{i j}(x, y)$.

Remark 2.2. A complete Finsler geometry model $\left(F:{ }_{F} \mathbf{N},{ }_{F} \mathbf{g},{ }_{F} \mathbf{D}\right)$ can be defined by additional assumptions on how three fundamental geometric objects (the $N$-connection ${ }_{F} \mathbf{N}$, the total metric ${ }_{F} \mathbf{g}$, the $d$-connection ${ }_{F} \mathbf{D}$ ) can be determined uniquely by a fundamental Finsler function $F$.

Finsler like geometries can be elaborated on a generic nonholonomic bundle/manifold $\mathbf{V}$ following self-consistent geometric and physically important principles (for instance, $\mathbf{V}=T M, \mathbf{V}$ is a (pseudo) Riemannian manifold with nonholonomic $2+2$ splitting [5]; there were performed similar generalizations for supermanifolds/superbundles and/or noncommutative generalizations, affine-Finsler spaces etc, see [25, 26, 14]).

2.2.2. The canonical Finsler connections and lifts of metrics. Let us consider $L=F^{2}$ is considered as an effective regular Lagrangian on $T M$ and action integral $S(\tau)=\int_{0}^{1} L(x(\tau), y(\tau)) d \tau$, for $y^{k}(\tau)=d x^{k}(\tau) / d \tau$, where $x(\tau)$ parameterizes smooth curves on a manifold $M$ with $\tau \in[0,1]$.

Lemma 2.1. The Euler-Lagrange equations $\frac{d}{d \tau} \frac{\partial L}{\partial y^{i}}-\frac{\partial L}{\partial x^{i}}=0$ are equivalent to the "nonlinear geodesic" (equivalently, semi-spray) equations $\frac{d^{2} x^{k}}{d \tau^{2}}+$ $2 \tilde{G}^{k}(x, y)=0$, where

$$
\tilde{G}^{k}=\frac{1}{4} \tilde{g}^{k j}\left(y^{i} \frac{\partial^{2} L}{\partial y^{j} \partial x^{i}}-\frac{\partial L}{\partial x^{j}}\right),
$$

for $\tilde{g}^{k j}$ being inverse to ${ }^{v} \tilde{g}_{i j} \equiv \tilde{g}_{i j}(25)$.

Certain geometric properties of fundamental Finsler functions can be studied via semi-spray configurations not concerning the problem of definition of connections and metrics for such spaces. For instance, J. Kern [36] suggested to consider nonhomomgeneous regular Lagrangians instead of those considered in Finsler geometry. That resulted in so-called Lagrange-Finsler geometry, on applications in modern physics see [19, 28].

Definition and Corollary 2.7. There is a canonical $N$-connection $\tilde{\mathbf{N}}=\left\{\tilde{N}_{j}^{a}\right\}$,

$$
\tilde{N}_{j}^{a}:=\frac{\partial \tilde{G}^{a}(x, y)}{\partial y^{j}},
$$

completely defined by the fundamental Finsler function $F$.

Proof. Using the above Lemma and local computations we can verify that the conditions Definition-Theorem (2.2) for $\mathrm{N}$-connections are satisfied. See also details of such a proof in [14]. 
We note that via $\tilde{\mathbf{N}}$ a Finsler metric $F$ defines naturally certain Nadapted frame structures $\tilde{\mathbf{e}}_{\nu}=\left(\tilde{\mathbf{e}}_{i}, e_{a}\right)$ and $\tilde{\mathbf{e}}^{\mu}=\left(e^{i}, \tilde{\mathbf{e}}^{a}\right)$ : we have to substitute $N_{j}^{a} \rightarrow \tilde{N}_{j}^{a}$ into, respectively, (8) and (9).

Definition and Corollary 2.8. A total metric structure on TM can be defined by a Sasaky type lift of $\tilde{g}_{i j}$,

$$
\tilde{\mathbf{g}}=\tilde{g}_{i j}(x, y) e^{i} \otimes e^{j}+\tilde{g}_{i j}(x, y) \tilde{\mathbf{e}}^{i} \otimes \tilde{\mathbf{e}}^{j} .
$$

It is possible to use other geometric principles for "lifts and projections" when, for instance, from a given $F$ it is constructed a complete homogeneous metric on total/horizontal spaces of $T M$. For models of locally anisotropic/Finsler gravity on $T M$, or on $\mathbf{V}$, a generalized covariance principle has to be considered following geometric and physical considerations [41]. Such constructions are performed up to certain frame/coordinate transforms $\tilde{\mathbf{e}}_{\gamma} \rightarrow \mathbf{e}_{\gamma^{\prime}}=e_{\gamma^{\prime}}^{\gamma} \tilde{\mathbf{e}}_{\gamma}$. From a formal point of view, we can omit "tilde" on symbols and write, in general, $\mathbf{g}=\left\{\mathbf{g}_{\alpha \beta}\right.$ and $\mathbf{N}=\left\{N_{i}^{a}=e^{a}{ }_{a^{\prime}} e_{i}{ }^{i^{\prime}} N_{i^{\prime}}^{a^{\prime}}\right\}$. We can define a subclass of frame/coordinate transforms preserving a prescribed splitting (1).

2.2.3. Models of Finsler-Cartan spaces. Using last two Definition-Corollaries, we prove

Theorem 2.5. A fundamental Finsler function $F(x, y)$ defines naturally a nonholonomic Riemann-Cartan model on $\widetilde{T M}$ determined by geometric data $\left(F:{ }_{F} \mathbf{N}=\tilde{\mathbf{N}},{ }_{F} \mathbf{g}=\tilde{\mathbf{g}},{ }_{F} \mathbf{D}=\mathbf{D}\right)$, where $\mathbf{D}$ is determined by $N$-adapted coefficients $\boldsymbol{\Gamma}_{\alpha \beta}^{\gamma}=\left(L_{j k}^{i}, L_{b k}^{a}, C_{j c}^{i}, C_{b c}^{a}\right)$ computed using formulas (16) for $\mathbf{g} \rightarrow \tilde{\mathbf{g}}(28)$ and $\mathbf{N} \rightarrow \tilde{\mathbf{N}}(27)$.

Introducing coefficients $\boldsymbol{\Gamma}_{\alpha \beta}^{\gamma}$, respectively, into formulas (17) and (18), we compute the torsion $\mathcal{T}$ and curvature $\mathcal{R}$ of $\mathbf{D}$.

In Finsler geometry it is largely used the Cartan d-connection $\tilde{\mathbf{D}}$ [23], see details in [9], which is also metric compatible and can be related to $\mathbf{D}$ (16) via frame transforms and deformations. If we consider that $L_{b k}^{a} \rightarrow L_{j k}^{i}$ and $C_{j c}^{i} \rightarrow C_{b c}^{a}$ for arbitrary $\mathbf{g}$ and $\mathbf{N}$ on $T M$ (i.e. we identify respectively $a=n+i$ with $i$ and $b=n+j)$, we obtain the so-called normal d-connection ${ }^{n} \mathbf{D}=\left({ }^{n} L^{i}{ }_{j k}, \quad{ }^{n} C_{j c}^{i}\right)$ where

$$
{ }^{n} L^{i}{ }_{j k}=\frac{1}{2} g^{i h}\left(\mathbf{e}_{k} g_{j h}+\mathbf{e}_{j} g_{k h}-\mathbf{e}_{h} g_{j k}\right), \quad{ }^{n} C_{b c}^{a}=\frac{1}{2} g^{a e}\left(e_{b} h_{e c}+e_{c} h_{e b}-e_{e} h_{b c}\right) .
$$

Definition 2.9. The Cartan d-connection $\tilde{\mathbf{D}}=\left(\tilde{L}^{i}{ }_{j k}, \tilde{C}_{j c}^{i}\right)$ is defined by introducing $\mathbf{g}=\tilde{\mathbf{g}}$ with $\tilde{h}_{i j}=\tilde{g}_{i j}$ and $\mathbf{N}=\tilde{\mathbf{N}}$ in (29).

Using formulas (17) and (18) for N-adapted coefficients of $\tilde{\mathbf{D}}$, we prove Theorem 2.6. The nontrivial components of torsion $\tilde{\mathbf{T}}_{\beta \gamma}^{\alpha}=\left\{\tilde{T}_{j c}^{i}, \tilde{T}_{i j}^{a}, \tilde{T}_{i b}^{a}\right\}$ and curvature $\tilde{\mathbf{R}}_{\beta \gamma \tau}^{\alpha}=\left\{\tilde{R}_{h j k}^{i}, \tilde{P}_{j k a}^{i}, \tilde{S}_{b c d}^{a}\right\}$ of $\tilde{\mathbf{D}}$ are respectively

$$
\tilde{T}_{j k}^{i}=0, \tilde{T}_{j c}^{i}=\tilde{C}_{j c}^{i}, \tilde{T}_{i j}^{a}=\tilde{\Omega}_{i j}^{a}, \tilde{T}_{i b}^{a}=e_{b}\left(\tilde{N}_{i}^{a}\right)-\tilde{L}_{b i}^{a}, \tilde{T}_{b c}^{a}=0,
$$


and

$$
\begin{aligned}
\tilde{R}_{h j k}^{i} & =\tilde{\mathbf{e}}_{k} \tilde{L}_{h j}^{i}-\tilde{\mathbf{e}}_{j} \tilde{L}_{h k}^{i}+\tilde{L}_{h j}^{m} \tilde{L}_{m k}^{i}-\tilde{L}_{h k}^{m} \tilde{L}^{i}{ }_{m j}-\tilde{C}_{h a}^{i} \tilde{\Omega}^{a}{ }_{k j}, \\
\tilde{P}^{i}{ }_{j k a} & =e_{a} \tilde{L}^{i}{ }_{j k}-\tilde{\mathbf{D}}_{k} \tilde{C}^{i}{ }_{j a}, \quad \tilde{S}_{b c d}^{a}=e_{d} \tilde{C}_{b c}^{a}-e_{c} \tilde{C}_{b d}^{a}+\tilde{C}_{b c}^{e} \tilde{C}^{a}{ }_{e d}-\tilde{C}_{b d}^{e} \tilde{C}^{a}{ }_{e c} .
\end{aligned}
$$

A very important property of $\left(\tilde{\mathbf{g}} ; \tilde{h}_{i j}=\tilde{g}_{i j}, \tilde{\mathbf{D}}\right)$ is that such geometric data can be encoded equivalently into an almost Kähler structure [10]. This allows us to perform deformation quantization and or A-brane quantization of Finsler geometry and generalizations, see [7, 6]. Such constructions are important for definition of almost Kähler spinors and Dirac operators in Finsler geometry (we do not present details in this work but emphasize that almost symplectic Finsler structures can be encoded into corresponding spinor and twistor structures).

2.2.4. On metric noncompatible Finsler geometries. Mathematicians elaborated different models of Finsler geometry generated by a fundamental Finsler function $F(x, y)$. Most known are constructions due to L. Berwald [42] and S. Chern [43] (see details in [13]) and "nonstandard" definition for the Ricci curvature by H. Akbar-Zadeh [37]. For instance,

- the Berwald d-connection is ${ }^{B} \mathbf{D}:=\left({ }^{B} L^{i}{ }_{j k}=\partial \tilde{N}_{j}^{i} / \partial y^{k},{ }^{B} C_{j c}^{i}=0\right)$;

- the Chern d-connection is ${ }^{C h} \mathbf{D}:=\left({ }^{C h} L^{i}{ }_{j k}=\tilde{L}^{i}{ }_{j k},{ }^{C h} C_{j c}^{i}=0\right)$.

The Chern's d-connection is very similar to the Levi-Civita connection, for geometric constructions on the $h$-subspace. The Finsler geometries determined by such $\mathrm{d}$-connections are not metric compatible on total space of $T M$ and characterized by nontrivial nonmetricity fields, $\mathcal{Q}:=\mathbf{D g},{ }^{B} \mathcal{Q} \neq 0$ and ${ }^{C h} \mathcal{Q} \neq 0$. We studied various generalizations affine-Finsler and affineLagrange spaces in Part I of [14]. Nontrivial nonmetricity fields (and "nonstandard" definitions of scalar and Ricci curvatures of Finsler spaces) present, in general, difficulties for definition of spinors and Dirac type operators, formulating conservation laws etc, see critical remarks in [28, 19]. So, there are substantial geometric and physical reasons to work with Finsler-Cartan type spaces and similar metric compatible configurations for applications in modern gravity and cosmology.

2.2.5. Finsler variables in general relativity. In this section, we show how the Einstein gravity can re-written equivalently in Finsler like variables.

Let us consider a (pseudo) Riemannian space $\mathbf{V}$ with nonholonomic $2+2$ splitting $\mathbf{N}=\left\{N_{i}^{a}\right\}$ and d-metric $\mathbf{g}=\left\{\mathbf{g}_{\alpha \beta}\right\}=\left\{\underline{g}_{\alpha^{\prime} \beta^{\prime}}\right\}$, which can be written in the form (13) and/or (14) and (15). We can always introduce on a welldefined cart for an atlas covering $\mathbf{V}$ a homogeneous function $\mathcal{F}(x, y)$ satisfying the conditions of Definition 2.6 and Remark 2.1. Using such a formal (pseudo) Finsler generating function, we can construct a Sasaki d-metric of type (28), for $\widetilde{f}_{i j}:=\frac{1}{2} \frac{\partial^{2} \mathcal{F}^{2}}{\partial y^{i} \partial y^{j}}$ and $\tilde{\mathcal{N}}_{j}^{a}$ obtained for $F \rightarrow \mathcal{F}$ following formulas $(27)$ and (26). With respect to dual local basis $d u^{\alpha}=\left(d x^{i}, d y^{a}\right)$, such a total metric 
can be written in the form

$$
\underline{f}_{\alpha \beta}=\left[\begin{array}{cc}
\widetilde{f}_{i j}+\widetilde{\mathcal{N}}_{i}^{a} \widetilde{\mathcal{N}}_{j}^{b} \widetilde{f}_{a b} & \widetilde{\mathcal{N}}_{j}^{e} \widetilde{f}_{a e} \\
\widetilde{\mathcal{N}}_{i}^{e} \widetilde{f}_{b e} & \widetilde{f}_{a b}
\end{array}\right] .
$$

Solving a quadratic algebraic equation for $e_{\alpha^{\prime}}^{\alpha}(u)$, for given values $\underline{g}_{\alpha^{\prime} \beta^{\prime}}$ and $\underline{f}_{\alpha \beta}(u)$,

$$
\underline{g}_{\alpha^{\prime} \beta^{\prime}}(u)=e_{\alpha^{\prime}}^{\alpha}(u) e_{\beta^{\prime}}^{\beta}(u) \underline{f}_{\alpha \beta}(u),
$$

we can re-write connections and tensors on $\mathbf{V}$, up to frame/coordinate transforms, in terms of variables $(\mathcal{F}: \widetilde{\mathbf{f}})$ or $(\mathbf{g}, \mathbf{N})$. We may change the carts and coordinates and $\mathcal{F}$ in order to get real well-defined solutions for vierbeins $e^{\alpha}{ }_{\alpha^{\prime}}$.

The above constructions depend on arbitrary generating function $\mathcal{F}$, which states a $2+2$ splitting via formulas $(27)$ and $(26)$ and respective frames (8) and (9), in their turn admitting transforms to N-elongated values determined by $N_{i}^{a}$ and/or $\widetilde{\mathcal{N}}_{j}^{a}$. This reflects the principle of general covariance when some additional nonholonomic constraints are imposed on frame structure. If a relation (32) is established on $\mathbf{V}$, we can compute the Levi-Civita connection $\nabla$ using the values $\underline{f}_{\alpha \beta}$ and/or, equivalently, $\mathbf{g}_{\alpha \beta}$. We can also compute the coefficients of $\mathbf{D}(16)$ and $\widetilde{\mathbf{D}}$ (29) with distortion relations of type (5). All such values are completely determined by $\underline{g}_{\alpha^{\prime} \beta^{\prime}}$ (equivalently by $\left.\underline{f}_{\alpha \beta}\right)$. Technically, it is difficult to solve in general form the Einstein equations of $\nabla$ written in Finsler like variable because they contain terms up to forth derivatives of $\mathcal{F}$ etc. Nevertheless, we can use some convenient data $(\mathbf{g}, \mathbf{N})$ in order to find a general solution $\mathbf{g}_{\alpha \beta}$ of the system (21) and to find some variables $\underline{f}_{\alpha \beta}$ using (32). If the constraints (22) are imposed additionally, we generate solutions in GR. For Finsler generalizations, we do not have to consider such Levi-Civita conditions.

Conclusion 2.1. $\quad$ 1. Any metric compatible Finsler-Cartan geometry can be modelled as a nonholonomic Riemann-Cartan geometry with an effective $d$-torsion completely determined by the metric and $N$-connection structures. We do not need additional algebraic equations as in EinsteinCartan gravity in order to find the d-torsion coefficients.

2. Any (pseudo) Riemannian manifold can be equivalently described by geometric data $(\mathbf{g}, \nabla)$, and/or $(\mathbf{g}, \mathbf{N}, \mathbf{D})$, and/or, in Finsler like variables, $\left(\mathcal{F}: \widetilde{\mathbf{f}}=\mathbf{g}, \widetilde{\mathcal{N}}_{j}^{a}, \widetilde{\mathbf{D}}\right)$.

\subsection{Conformal transforms and $\mathrm{N}-$ connections}

With respect to arbitrary or coordinate frames, it is not a trivial task to define conformal transforms because of generic anisotropy of spaces enabled with $\mathrm{N}-$ connection structure (in particular, for Finsler-Cartan spaces) and nonlinear dependence of metric and connections on $N_{i}^{a}$ and/or $\widetilde{\mathcal{N}}_{j}^{a}$. Nevertheless, in $\mathrm{N}$-adapted frames (8) and (9), certain analogy to Riemann-Cartan spaces can be found. 
Let us denote by $\mathbf{D}$ any of metric compatible d-connections (16) or $\tilde{\mathbf{D}}$ (29). The torsion and curvature tensors (see $\mathrm{N}$-adapted coefficients (17) and (18) and, respectively, (30) and (31)) are computed in abstract index form via

$$
\boldsymbol{\Delta}_{\alpha \beta} f=\mathbf{T}_{\alpha \beta}^{\gamma} \mathbf{D}_{\gamma} f \text { and }\left(\boldsymbol{\Delta}_{\alpha \beta}-\mathbf{T}_{\alpha \beta}^{\gamma} \mathbf{D}_{\gamma}\right) \mathbf{V}^{\tau}=\mathbf{R}_{\alpha \beta \gamma}^{\tau} \mathbf{V}^{\gamma}
$$

for

$$
\boldsymbol{\Delta}_{\alpha \beta}:=\mathbf{D}_{\alpha} \mathbf{D}_{\beta}-\mathbf{D}_{\beta} \mathbf{D}_{\alpha}=2 \mathbf{D}_{[\alpha} \mathbf{D}_{\beta]}
$$

and arbitrary scalar function $f(x, y)$ and $\mathrm{d}$-vector $\mathbf{V}^{\gamma}$ (in this work, we follow a different rule/order of contracting indices than that in [1]).

We can consider a source d-tensor $\Upsilon_{\alpha \beta}=-\lambda \mathbf{g}_{\alpha \beta}+8 \pi G \mathbf{T}_{\alpha \beta}$, where, for $2+2$ splitting, $\lambda$ and $G$ are respectively the cosmological and Newton constants (such values can be defined via Sasaki lifts, for $4+4$ models on tangent bundles). The Einstein equations for $\mathbf{D}_{\alpha}$ can be written similarly to $(21)$,

$$
\mathbf{R}_{\alpha \beta}-\frac{1}{2} \mathbf{g}_{\alpha \beta}{ }_{s} R+\lambda \mathbf{g}_{\alpha \beta}=8 \pi G \mathbf{T}_{\alpha \beta},
$$

where $\mathbf{R}_{\alpha \beta}:=\mathbf{R}_{\alpha \beta \gamma}^{\gamma}$ and ${ }_{s} R:=\mathbf{g}^{\alpha \beta} \mathbf{R}_{\alpha \beta}$. In the spinor formulation of gravity, there are used

$$
\begin{aligned}
{ }_{s} R & :=24 \Lambda=4 \lambda-8 \pi G \mathbf{T}_{\tau}^{\tau}, \\
\boldsymbol{\Phi}_{\alpha \beta} & :=3 \Lambda \mathbf{g}_{\alpha \beta}-\frac{1}{2} \mathbf{R}_{\alpha \beta}=8 \pi G\left(\frac{1}{4} \mathbf{T}_{\tau}^{\tau} \mathbf{g}_{\alpha \beta}-\mathbf{T}_{\alpha \beta}\right)
\end{aligned}
$$

and the conformal d-tensor

$$
\mathbf{C}_{\alpha \beta}^{\tau}{ }^{\gamma}:=\mathbf{R}_{\alpha \beta}^{\tau}{ }^{\gamma}+2 \mathbf{R}_{[\alpha}{ }^{[\tau} \delta_{\beta]}^{\gamma]}+\frac{1}{3}{ }_{s} R \delta_{[\alpha}^{\gamma} \delta_{\beta]}^{\tau}=\mathbf{R}_{\alpha \beta}^{\tau}{ }^{\gamma}+4 \mathbf{P}_{[\alpha}{ }^{[\tau} \delta_{\beta]}^{\gamma]}
$$

where $\delta_{\beta}^{\gamma}$ is the Kronecker symbol and

$$
2 \mathbf{P}_{\alpha \beta}=\frac{1}{6}{ }_{s} R \mathbf{g}_{\alpha \beta}-\mathbf{R}_{\alpha \beta} .
$$

Such d-tensor formulas are related to similar ones for the Levi-Civita connection $\nabla$ via distortions $\mathbf{D}=\nabla+\mathbf{Q}(5)$, where all values are determined by a corresponding $\mathrm{d}$-metric (13) or (28). This results in distortions of d-tensors,

$$
\begin{aligned}
\mathbf{R}_{\alpha \beta} & =R_{\alpha \beta}+\mathbf{Q}_{\alpha \beta},{ }_{s} R=R+{ }_{s} Q, \\
\mathbf{R}_{\tau \alpha \beta \gamma} & =R_{\tau \alpha \beta \gamma}+\mathbf{Q}_{\tau \alpha \beta \gamma}, \mathbf{C}_{\tau \alpha \beta \gamma}=C_{\tau \alpha \beta \gamma}+{ }_{W} \mathbf{Q}_{\tau \alpha \beta \gamma},
\end{aligned}
$$

were the left label ${ }_{W} \mathbf{Q}_{\tau \alpha \beta \gamma}$ is from the distortion of Weyl's type conformal d-tensor.

Proposition 2.3. Under conformal transforms of coefficients d-metric (13),

$$
\widehat{\mathbf{g}}_{\alpha \beta}:=\varpi^{2}(u) \mathbf{g}_{\alpha \beta},
$$

preserving the $N$-connection structure $\mathbf{N}=\left\{N_{i}^{a}\right\}$, the conformal $d$-tensor (36) satisfies the conditions

$$
\widehat{\mathbf{C}}_{\tau \alpha \beta \gamma}=\varpi^{2} \mathbf{C}_{\tau \alpha \beta \gamma} \text { and } \widehat{\mathbf{C}}_{\alpha \beta \gamma}^{\tau}=\mathbf{C}_{\alpha \beta \gamma}^{\tau} .
$$


Proof. Such transforms can be verified by a N-adapted calculus with respect to fixed $\mathrm{N}$-elongated (8) and (9). We note here that with respect to a coordinate frame, for a metric (14) with coefficients (15), a transform (39) define a nonlinear transform of metric. The property of rescalling holds only for the d-metric coefficients with respect to fixed data $\mathbf{N}=\left\{N_{i}^{a}\right\}$.

The Bianchi identities for $\mathbf{D}$,

$$
\mathbf{D}_{[\alpha} \mathbf{R}_{\tau \alpha] \beta \gamma}=0, \text { or } \mathbf{D}^{\tau} \widehat{\mathbf{C}}_{\gamma \tau \alpha \beta}=-2 \mathbf{D}_{[\beta} \mathbf{P}_{\gamma] \alpha}
$$

are standard ones with possible $h$ - and $v$-projections [14].

Theorem 2.7. For any fixed data $\left(\mathbf{g}_{\alpha \beta}, N_{i}^{a}\right)$, there is a nonholonomic deformation to some $\left(\mathbf{g}_{\alpha^{\prime} \beta^{\prime}}, N_{i^{\prime}}^{a^{\prime}}\right)$ for which $\mathbf{C}_{\tau^{\prime} \alpha^{\prime} \beta^{\prime} \gamma^{\prime}}=0$ with respect to $a$ re-defined $\mathbf{e}^{\beta^{\prime}}=\left(e^{j^{\prime}}, \mathbf{e}^{b^{\prime}}\right)(9)$.

Proof. Let us fix a d-metric (13) with coefficients $\mathbf{g}_{\alpha^{\prime} \beta^{\prime}}:=\varpi^{2}(u) \eta_{\alpha \beta}$ with $\eta_{\alpha \beta}$ being diagonal constants of any necessary signature $( \pm 1, \pm, \ldots, \pm)$, with respect to some $\mathbf{e}^{\beta^{\prime}}=\left(e^{j^{\prime}}=d x^{j^{\prime}}, \mathbf{e}^{b^{\prime}}=d y^{b^{\prime}}+N_{i^{\prime}}^{b^{\prime}} d x^{i^{\prime}}\right)$. For such a $\mathrm{d}$-metric and $\mathrm{N}$-adapted co-bases, we can verify that $\mathbf{C}_{\tau^{\prime} \alpha^{\prime} \beta^{\prime} \gamma^{\prime}}=0$, as a consequence of Proposition 2.3. We can redefine data $\left((13), \mathbf{g}_{\alpha^{\prime} \beta^{\prime}}\right)$ in a coordinate form (14) with coefficients $(15)$ (with primed indices, $g_{\underline{\alpha}^{\prime} \underline{\beta}^{\prime}}$ ). Then considering arbitrary frame transforms $e^{\underline{\alpha}^{\prime}}$ we can compute $g_{\underline{\alpha} \underline{\beta}}=e^{\underline{\alpha}^{\prime}} e^{\underline{\beta}^{\prime}} g_{\underline{\alpha^{\prime}}} \underline{\beta}^{\prime}$. Finally, we can redefine for a nonholonomic $2+2$, or $4+4$, splitting certain data $\left(\mathbf{g}_{\alpha \beta}, N_{i}^{a}\right)$, for which, in general, $\mathbf{C}_{\tau \alpha \beta \gamma} \neq 0$, and the corresponding to $\nabla, C_{\tau \alpha \beta \gamma} \neq 0$. Such constructions with nonholonomic deformations are possible because vierbeins (11) may depend on some $N$-coefficients which can be present also in the generic off-diagonal form of "primary" metric. The transformation laws of $\mathrm{d}$-objects on nonholonomic manifolds with $\mathrm{N}$-connection are different from those on usual manifolds without $\mathrm{N}$-connection splitting (1).

Conclusion 2.2. An arbitrary (pseudo) Riemannian spacetime $V$ with metric structure $\mathbf{g}=\left\{g_{\alpha \beta}\right\}$ is not conformally flat, i.e. $C_{\tau \alpha \beta \gamma} \neq 0$, for $\nabla$. Nevertheless, we can always associate a nonholonomic manifold $\mathbf{V}$ enabled with the same metric structure but with such a $N$-connection $\mathbf{N}$ when the corresponding canonical d-connection $\mathbf{D}$ is with zero Weyl d-tensor $\mathbf{C}_{\tau \alpha \beta \gamma}=0$ (we omit priming of indices).

The above values $C_{\tau \alpha \beta \gamma}$ and $\mathbf{C}_{\tau \alpha \beta \gamma}$ are related in unique form by distortions (5) for a unique $\mathbf{D}=\nabla+\mathbf{Q}$ (5). Such constructions depend on prescribed distribution $\mathbf{N}$. They do not violate a principle of general covariance on $V$, or $\mathbf{V}$. We can prescribe a necessary type distribution, adapted all constructions to $\mathrm{N}$-splitting, and then re-define everything in arbitrary systems of reference.

Finally, we note that similar statements can be formulated, up to some frame transforms $(32)$, for the cases when $(\mathbf{g}, \mathbf{N}, \mathbf{D}) \rightarrow(\tilde{\mathbf{g}}, \tilde{\mathbf{N}}, \tilde{\mathbf{D}})$, i.e. for a Finsler-Cartan space, or any such variables on a (pseudo) Riemannian manifold. 


\section{Finsler-Cartan Spinors and Einstein Gravity}

Spinor and twistor geometries for data $(\mathbf{g}, \mathbf{N}, \mathbf{D})$ and $\mathrm{N}$-adapted frames can be elaborated $[20,21,22,26,14]$ similarly to those for $(\mathbf{g}, \nabla)$ in arbitrary frames of reference $[1,2,3,4]$. The concept of distinguished spinor, $\mathrm{d}$-spinor, was introduced as a couple of $h$ - and $v$-spinors derived for a $\mathrm{N}$-connection splitting (1), see a brief summary in sections 2.2 and 3.1 of [26].

\subsection{Spinors and $\mathrm{N}$-connections}

We provide main definitions and introduce an abstract index formalism adapted nonholonomic manifolds/bundles with $n+m$ splitting

\subsubsection{Clifford $\mathrm{N}$-adapted structures and spin $\mathrm{d}$-connections.}

Definition 3.1. We define a Clifford $d$-algebra as a $\wedge V^{n+m}$ algebra determined by a product $\mathbf{u v}+\mathbf{v u}=2 \mathbf{g}(\mathbf{u}, \mathbf{v}) \mathbb{I}$, with associated $h-$, $v$-products

$$
{ }^{h} u{ }^{h} v+{ }^{h} v{ }^{h} u=2{ }^{h} g(u, v){ }^{h} \mathbb{I},{ }^{v} u{ }^{v} v+{ }^{v} v{ }^{v} u=2{ }^{v} h\left({ }^{v} u, \quad{ }^{v} v\right){ }^{v} \mathbb{I},
$$

for any $\mathbf{u}=\left({ }^{h} u,{ }^{v} u\right), \mathbf{v}=\left({ }^{h} v,{ }^{v} v\right) \in V^{n+m}$, where $\mathbb{I},{ }^{h} \mathbb{I}$ and ${ }^{v} \mathbb{I}$ are unity matrices of corresponding dimensions $(n+m) \times(n+m)$, or $n \times n$ and $m \times m .^{7}$

Any metric ${ }^{h} g$ on $h \mathbf{V}$ is defined by sections of $T h \mathbf{V}$ provided with a bilinear symmetric form on continuous sections $\operatorname{Sec}(T h \mathbf{V})$. We can define Clifford h-algebras ${ }^{h} \mathcal{C} l\left(T_{x} h \mathbf{V}\right), \gamma_{i} \gamma_{j}+\gamma_{j} \gamma_{i}=2 g_{i j}{ }^{h} \mathbb{I}$, in any point $x \in T h \mathbf{V}$.

The Clifford $\mathrm{d}-$ module of a vector bundle $E$ (in general, we can consider a complex vector bundle ${ }^{E} \pi: E \rightarrow \mathbf{V}$ ) is defined by the $C(\mathbf{V})$-module $\operatorname{Sec}(E)$ of continuous sections in $E, c: \operatorname{Sec}\left({ }^{N} \mathcal{C l}(\mathbf{V})\right) \rightarrow \operatorname{End}(\operatorname{Sec}(E))$. Prescribing a $\mathrm{N}$-connection structure, a Clifford $\mathrm{N}$-anholonomic bundle on $\mathbf{V}$ is by definition ${ }^{N} \mathcal{C l}(\mathbf{V}) \doteq{ }^{N} \mathcal{C l}\left(T^{*} \mathbf{V}\right)$, where $T^{*}$ is the dual tangent bundle.

Definition 3.2. A Clifford d-space associated to data $\mathbf{g}(x, y)$ (13) and $\mathbf{N}$ for a nonholonomic manifold $\mathbf{V}$ is defined as a Clifford bundle

$$
\mathcal{C l}(\mathbf{V})={ }^{h} \mathcal{C l}(h \mathbf{V}) \oplus{ }^{v} \mathcal{C l}(v \mathbf{V}),
$$

with Clifford $h$-space, ${ }^{h} \mathcal{C} l(h \mathbf{V}) \doteq{ }^{h} \mathcal{C} l\left(T^{*} h \mathbf{V}\right)$, and Clifford $v$-space, ${ }^{v} \mathcal{C} l(v \mathbf{V}) \doteq{ }^{v} \mathcal{C l}\left(T^{*} v \mathbf{V}\right)$.

Let $V^{n}$ be a vector space provided with Clifford structure. We write ${ }^{h} V^{n}$ if its tangent space is provided with a quadratic form ${ }^{h} g$ and consider ${ }^{h} \mathcal{C l}\left(V^{n}\right) \equiv \mathcal{C l}\left({ }^{h} V^{n}\right)$ using the subgroup $S O\left({ }^{h} V^{n}\right) \subset O\left({ }^{h} V^{n}\right)$. A standard definition of spinors is possible using sections of a vector bundle $S$ on a manifold $M$ being considered an irreducible representation of the group $\operatorname{Spin}(M) \doteq \operatorname{Spin}\left(T_{x}^{*} M\right)$ defined on the typical fiber. The set of sections $\operatorname{Sec}(S)$ defines an irreducible Clifford module.

\footnotetext{
7 in certain cases, we shall consider only "horizontal" geometric constructions if they are similar to "vertical" ones
} 
The space of complex $\mathrm{h}$-spins is defined by the subgroup

$$
{ }^{h} \operatorname{Spin}^{c}(n) \equiv \operatorname{Spin}^{c}\left({ }^{h} V^{n}\right) \equiv{ }^{h} \operatorname{Spin}^{c}\left(V^{n}\right) \subset \mathcal{C l}\left({ }^{h} V^{n}\right),
$$

determined by the products of pairs of vectors $w \in{ }^{h} V^{\mathbb{C}}$ when $w \doteq p u$ where $p$ is a complex number of module 1 and $u$ is of unity length in ${ }^{h} V^{n}$. Similar constructions can be performed for the $\mathrm{v}$-subspace ${ }^{v} V^{m}$, which allows us to define similarly the group of real v-spins. A h-spinor bundle ${ }^{h} S$ on a h-space $h \mathbf{V}$ is a complex vector bundle with both defined action of the $\mathrm{h}-$ spin group ${ }^{h} \operatorname{Spin}\left(V^{n}\right)$ on the typical fiber and an irreducible representation of the group ${ }^{h} \operatorname{Spin}(\mathbf{V}) \equiv \operatorname{Spin}(h \mathbf{V}) \doteq \operatorname{Spin}\left(T_{x}^{*} h \mathbf{V}\right)$. The set of sections $\operatorname{Sec}\left({ }^{h} S\right)$ defines an irreducible Clifford h-module.

Definition 3.3. A distinguished spinor (d-spinor) bundle $\mathbf{S} \doteq\left({ }^{h} S,{ }^{v} S\right)$ for $\mathbf{V}, \operatorname{dim} \mathbf{V}=n+m$, is a complex vector bundle with an action of the spin distinguished (d-group) Spin $\mathbf{V} \doteq \operatorname{Spin}\left(V^{n}\right) \oplus \operatorname{Spin}\left(V^{m}\right)$ with an irreducible representation $\operatorname{Spin}(\mathbf{V}) \doteq \operatorname{Spin}\left(T^{*} \mathbf{V}\right)$. The set of $\operatorname{sections} \operatorname{Sec}(\mathbf{S})=$ $\operatorname{Sec}\left({ }^{h} S\right) \oplus \operatorname{Sec}\left({ }^{v} S\right)$ is an irreducible Clifford d-module.

The considerations presented above provide a proof for

Theorem 3.1. Any d-metric and N-connection structures define naturally the fundamental geometric objects and structures (such as the Clifford $h-$ module, v-module and Clifford d-modules, or the h-spin, v-spin structures and $d$-spinors) for the corresponding nonholonomic spin manifold and/or $N$-anholonomic spinor (d-spinor) manifold.

We consider a Hilbert space of finite dimension and denote a local dual coordinate basis $e^{\underline{i}} \doteq d x^{\underline{i}}$ on $h \mathbf{V}$. In $\mathrm{N}$-adapted form, it is possible to introduce certain classes of orthonormalized vielbeins and the $\mathrm{N}$-adapted vielbeins, $e^{\hat{\imath}} \doteq e_{\underline{i}}^{\hat{\imath}}(x, y) e^{\underline{i}}$ and $e^{i} \doteq e_{\underline{i}}^{i}(x, y) e^{\underline{i}}$, when $g^{\underline{i} \underline{j}} e_{\underline{i}}^{\hat{\imath}} e^{\hat{\jmath}}{ }_{\underline{j}}=\delta^{\hat{\imath} \hat{\jmath}}$ and $g^{\underline{i} \underline{j}} e_{\underline{i}}^{i} \underline{e}^{j}{ }_{j}=g^{i j}$. This allows us to define the algebra of Dirac's gamma $\mathrm{h}-$ matrices with self-adjoint matrices $M_{k}(\mathbb{C})$, where $k=2^{n / 2}$ is the dimension of the irreducible representation of $\mathcal{C l}(h \mathbf{V})$ derived from the relation $\gamma^{\hat{\imath}} \gamma^{\hat{\jmath}}+\gamma^{\hat{\jmath}} \gamma^{\hat{\imath}}=2 \delta^{\hat{\imath} \hat{\jmath}} h \mathbb{I}$. The action of $d x^{i} \in \mathcal{C l}(h \mathbf{V})$ on a spinor ${ }^{h} \psi \in{ }^{h} S$ can be parameterized by formulas ${ }^{h} c\left(d x^{\hat{\imath}}\right) \doteq \gamma^{\hat{\imath}}$ and ${ }^{h} c\left(d x^{i}\right){ }^{h} \psi \doteq \gamma^{i}{ }^{h} \psi \equiv$ $e^{i}{ }_{\hat{\imath}} \gamma^{\hat{\imath}}{ }^{h} \psi$. The algebra of Dirac's gamma $\mathrm{v}$-matrices is defined by selfadjoint matrices $M_{k}^{\prime}(\mathbb{C})$, where $k^{\prime}=2^{m / 2}$ is the dimension of the irreducible representation of $\mathcal{C} l(F)$, for a typical fiber $F$ ), when $\gamma^{\hat{a}} \gamma^{\hat{b}}+\gamma^{\hat{b}} \gamma^{\hat{a}}=2 \delta^{\hat{a} \hat{b}}{ }^{v} \mathbb{I}$. The action of $d y^{a} \in \mathcal{C} l(F)$ on a spinor ${ }^{v} \psi \in{ }^{v} S$ is ${ }^{v} c\left(d y^{\hat{a}}\right) \doteq \gamma^{\hat{a}}$ and ${ }^{v} c\left(d y^{a}\right)^{v} \psi \doteq \gamma^{a}{ }^{v} \psi \equiv e_{\hat{a}}^{a} \gamma^{\hat{a}}{ }^{v} \psi$.

In general, a matrix calculus with gamma d-matrices can be elaborated for a total d-metric structure $\mathbf{g}={ }^{h} g \oplus{ }^{v} h$. We consider d-spinors $\breve{\psi} \doteq\left({ }^{h} \psi,{ }^{v} \psi\right) \in \mathbf{S} \doteq\left({ }^{h} S,{ }^{v} S\right)$ and d-gamma matrix relations $\gamma^{\hat{\alpha}} \gamma^{\hat{\beta}}+$ $\gamma^{\hat{\beta}} \gamma^{\hat{\alpha}}=2 \delta^{\hat{\alpha} \hat{\beta}} \mathbb{I}$. The action of $d u^{\alpha} \in \mathcal{C l}(\mathbf{V})$ on a d-spinor $\breve{\psi} \in \mathbf{S}$ resulting in distinguished irreducible representations $\mathbf{c}\left(d u^{\hat{\alpha}}\right) \doteq \gamma^{\hat{\alpha}}$ and

$$
\mathbf{c}=\left(d u^{\alpha}\right) \breve{\psi} \doteq \gamma^{\alpha} \breve{\psi} \equiv e_{\hat{\alpha}}^{\alpha} \gamma^{\hat{\alpha}} \breve{\psi} .
$$


We obtain d-metric - d-gamma matrix relations

$$
\gamma^{\alpha}(u) \gamma^{\beta}(u)+\gamma^{\beta}(u) \gamma^{\alpha}(u)=2 g^{\alpha \beta}(u) \mathbb{I},
$$

which can re-written for "boldface" coefficients of metric. In irreducible form $\breve{\gamma} \doteq{ }^{h} \gamma \oplus{ }^{v} \gamma$ and $\breve{\psi} \doteq{ }^{h} \psi \oplus{ }^{v} \psi$, or, $\gamma^{\alpha} \doteq\left({ }^{h} \gamma^{i},{ }^{v} \gamma^{a}\right)$ and $\breve{\psi} \doteq\left({ }^{h} \psi,{ }^{v} \psi\right)$

The spin connection ${ }_{S} \nabla$ for (pseudo) Riemannian manifolds is standardly determined by the Levi-Civita connection, ${ }_{S} \nabla \doteq d-\frac{1}{4} \Gamma^{i}{ }_{j k} \gamma_{i} \gamma^{j} d x^{k}$. Similar constructions are possible for nonholonomic manifolds enabled with metric compatible d-connections (for instance, in Finsler-Cartan geometry). The spin $\mathrm{d}$-connection operators $\mathbf{s} \nabla$ can be similarly constructed from any metric compatible d-connection $\Gamma_{\beta \mu}^{\alpha}$ (for instance, with coefficients (16), or (29)), when for a scalar function $f(x, y)$ in the form

$$
\delta f=\left(\mathbf{e}_{\nu} f\right) \delta u^{\nu}=\left(\mathbf{e}_{i} f\right) d x^{i}+\left(e_{a} f\right) \delta y^{a},
$$

for $\delta u^{\nu}=\mathbf{e}^{\nu}(9)$.

Definition 3.4. The canonical (Finsler-Cartan) spin $d$-connection is defined by $\mathbf{D}=\left\{\boldsymbol{\Gamma}_{\beta \mu}^{\alpha}\right\}\left(\tilde{\mathbf{D}}=\left\{\tilde{\boldsymbol{\Gamma}}_{\beta \mu}^{\alpha}\right\}\right)$ following formula:

$$
\mathbf{S} \mathbf{D} \doteq \delta-\frac{1}{4} \boldsymbol{\Gamma}_{\beta \mu}^{\alpha} \gamma_{\alpha} \gamma^{\beta} \mathbf{e}^{\mu}\left(\mathbf{s} \tilde{\mathbf{D}} \doteq \delta-\frac{1}{4} \tilde{\boldsymbol{\Gamma}}_{\beta \mu}^{\alpha} \gamma_{\alpha} \gamma^{\beta} \mathbf{e}^{\mu}\right)
$$

For the purposes of this work, we shall consider abstract index formulations of $\mathrm{d}-$-spinor calculus for nonholonomic manifolds/bundles with splitting $2+2$, or $4+4$.

3.1.2. Abstract $\mathbf{d}$-tensor and $\mathbf{d}$-spinor indices. Indices of $d$-tensors are considered as a set of labels which can changed into respective sets of $\mathrm{d}$-spinor indices, primed and unprimed (with dots and without dots) following, for instance, such rules: $\Psi_{\mu}^{\alpha \beta}=\Psi^{\dot{A} \dot{A}^{\prime} \dot{B} \dot{B}^{\prime}}{ }_{\dot{M} \dot{M}^{\prime}}$, where dot spinor capital indices correspond small Greek tensor indices. For $h$ - and/or $v$-decompositions, when $\xi^{\alpha}=\left(\xi^{i}, \xi^{a}\right)$, we shall write $\xi^{\dot{A} \dot{A}^{\prime}}=\left(\xi^{I I^{\prime}}, \xi^{A A^{\prime}}\right)$, where $\xi^{I I^{\prime}}$ is for a horizontal spinor-vector and $\xi^{A A^{\prime}}$ is for vertical spinor-vector. In similar forms, we can consider $h$ - and $v$ - and spinor decompositions for forms and tensors with mixed indices. So, we shall follow the formalism from [1] but re-defined in a form to be able to encode spinorially d-tensors with possible $\mathrm{N}$-adapted splitting. Primed spinor indices are complex conjugated with corresponding unprimed, for instance, $\overline{\xi^{\dot{A} \dot{A}^{\prime}}}=\bar{\xi}^{\dot{A}^{\prime} \dot{A}}, \overline{\xi^{I I^{\prime}}}=\bar{\xi}^{I^{\prime} I}$ etc both for up and low indices.

We can consider antisymmetric $\varepsilon$-spinors on total spaces and $h$ - and $v$-subspaces with the properties,

$$
\begin{aligned}
\varepsilon^{\dot{A}^{\prime} \dot{B}^{\prime}} & :=\bar{\varepsilon}^{\dot{A}^{\prime} \dot{B}^{\prime}}=\overline{\varepsilon^{\dot{A} \dot{B}}}, \varepsilon_{\dot{A}^{\prime} \dot{B}^{\prime}}:=\bar{\varepsilon}_{\dot{A}^{\prime} \dot{B}^{\prime}}=\overline{\varepsilon_{\dot{A} \dot{B}}} \\
\varepsilon^{I^{\prime} J^{\prime}} & :=\bar{\varepsilon}^{I^{\prime} J^{\prime}}=\overline{\varepsilon^{I J}}, \varepsilon_{I^{\prime} J^{\prime}}:=\bar{\varepsilon}_{I^{\prime} J^{\prime}}=\overline{\varepsilon_{I J}} \text { for h-spinor indices; } \\
\varepsilon^{A^{\prime} B^{\prime}}: & =\bar{\varepsilon}^{A^{\prime} B^{\prime}}=\overline{\varepsilon^{A B}}, \varepsilon_{A^{\prime} B^{\prime}}:=\bar{\varepsilon}_{A^{\prime} B^{\prime}}=\overline{\varepsilon_{A B}} \text { for v-spinor indices. }
\end{aligned}
$$

This is related to the rules of transforming low indices into up ones, and inversely, using metrics and/or d-metrics, for instance, $\mathbf{g}_{\alpha \beta}=\left[g_{i j}, g_{a b}\right]$ and 
$\mathbf{g}^{\alpha \beta}=\left[g^{i j}, g^{a b}\right]$. In brief, the spinor decompositions of metrics are written in the form,

$$
\begin{aligned}
\mathbf{g}_{\alpha \beta} & =\varepsilon_{\dot{A} \dot{B}^{A^{\prime} \dot{B}^{\prime}}}, \mathbf{g}^{\alpha \beta}=\varepsilon^{\dot{A} \dot{B}} \varepsilon^{\dot{A}^{\prime} \dot{B}^{\prime}} ; \\
g_{i j} & =\varepsilon_{I J} \varepsilon_{I^{\prime} J^{\prime}}, g^{i j}=\varepsilon^{I J} \varepsilon^{I^{\prime} J^{\prime}}, \text { for h-metrics; } \\
g_{a b} & =\varepsilon_{A B} \varepsilon_{A^{\prime} B^{\prime}}, g^{a b}=\varepsilon^{A B} \varepsilon^{A^{\prime} B^{\prime}}, \text { for v-metrics. }
\end{aligned}
$$

In our works $[20,21,22,26,14]$, we used also N-adapted gamma matrices generating corresponding Clifford algebras for spinors (some authors call them $\sigma$-symbols or transition indices from Minkowski tetrads to spin systems of reference, on corresponding tangent bundles). In brief, such a formalism is related to orthonormalized (co) bases, $\mathbf{e}_{\alpha^{\prime}}=\left(\mathbf{e}_{i^{\prime}}, e_{a^{\prime}}\right)$ and $\mathbf{e}^{\beta^{\prime}}=\left(e^{j^{\prime}}, \mathbf{e}^{b^{\prime}}\right)$, where $\mathrm{d}$-tensor primed indices are used for definition of $4 \times 4 \gamma$-matrices $\gamma_{\alpha^{\prime}}=\left(\gamma_{\alpha^{\prime}}^{\dot{A} \dot{A}^{\prime}}\right)$ satisfying the relations

$$
\gamma_{\alpha^{\prime}} \gamma_{\beta^{\prime}}+\gamma_{\beta^{\prime}} \gamma_{\alpha^{\prime}}=2 \eta_{\alpha^{\prime} \beta^{\prime}}
$$

where the Minkowski metric $\eta_{\alpha^{\prime} \beta^{\prime}}$ is, for instance, of signature $(+++-)$ for a formal $2+2$ splitting ${ }^{8}$. Using transforms of type $\mathbf{e}_{\alpha^{\prime}} \frac{\alpha}{}=\mathbf{e}_{\alpha^{\prime}}^{\alpha} \mathbf{e}_{\alpha}^{\alpha}$ and their inverse, we can write

$$
\gamma_{\alpha} \gamma_{\beta}+\gamma_{\beta} \gamma_{\alpha}=2 \mathbf{g}_{\alpha \beta} \text { and } \gamma_{\underline{\alpha}} \gamma_{\underline{\beta}}+\gamma_{\underline{\beta}} \gamma_{\underline{\alpha}}=2 \mathbf{g}_{\underline{\alpha}} \underline{\beta}
$$

where d-metric (13) and, respectively, (14) and (15), including $\mathrm{N}$-coefficients, are considered for $\mathbf{e}_{\alpha}=\mathbf{e}_{\alpha} \underline{\alpha} \partial_{\underline{\alpha}}$ and $\mathbf{e}^{\beta}=\mathbf{e}^{\beta}{ }_{\beta} d u^{\underline{\beta}}$ with decompositions of type (10) and (11). With $\gamma$-matrices, for instance the first relation in (41) is written

$$
\mathbf{g}_{\alpha \beta}=\gamma_{\alpha}^{\dot{A} \dot{A}^{\prime}} \gamma_{\beta}^{\dot{B} \dot{B}^{\prime}} \varepsilon_{\dot{A} \dot{B}} \varepsilon_{\dot{A}^{\prime} \dot{B}^{\prime}}
$$

for $\gamma_{\alpha^{\prime}}^{\dot{A} \dot{A}^{\prime}}:=\mathbf{e}_{\alpha^{\prime}}^{\alpha} \gamma_{\alpha}^{\dot{A} \dot{A}^{\prime}}$ (we omit similar decompositions for $h$ - and $v$-indices). For simplicity, in this work we shall follow abstract algebraic decompositions not writing gamma matrices even formulas of type (43) are necessary for constructing in explicit form exact generic off-diagonal solutions with nontrivial $\mathrm{N}$-coefficients of Einstein-Dirac systems.

\section{2. $\mathrm{N}$-adapted spinors and nonholonomic (Finsler) gravity}

3.2.1. $\mathrm{N}$-adapted covariant derivatives and spin coefficients. In brief, we shall write the $\mathrm{d}$-spinor equivalents as

$$
\mathbf{D}_{\alpha}=\mathbf{D}_{\dot{A} \dot{A}^{\prime}}=\mathbf{D}_{\dot{A}^{\prime} \dot{A}}, D_{i}=D_{I I^{\prime}}=D_{I^{\prime} I}, D_{a}=D_{A A^{\prime}}=D_{A^{\prime} A}
$$

etc. Fixing spin diads $\varepsilon_{\dot{A}}^{\dot{A}}=\left(\varepsilon_{\dot{A}}^{0}, \varepsilon_{\dot{A}}^{1}\right), \varepsilon_{I}^{I}=\left(\varepsilon_{I}^{0}, \varepsilon_{I}^{1}\right), \varepsilon_{\frac{A}{A}}=\left(\varepsilon_{A}^{0}, \varepsilon_{A}^{1}\right)$, and theirs respective duals, $\varepsilon_{\underline{\dot{A}}}^{\dot{A}}, \varepsilon_{\underline{I}}^{I}, \varepsilon_{\underline{A}}^{A},{ }^{9}$ we can introduce $\mathrm{N}$-adapted $\mathrm{d}$-spin

\footnotetext{
${ }^{8}$ on tangent bundles to Lorentz manifolds of dimension $8=4+4$, we can use one such a gamma relation for the $\mathrm{h}$-subspace and another one for the $\mathrm{v}$-subspace

9 in our approach the underlined indices are equivalent to "boldface" indices in [1]
} 
coefficients,

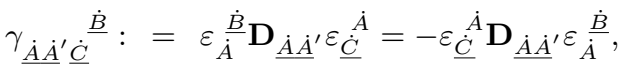

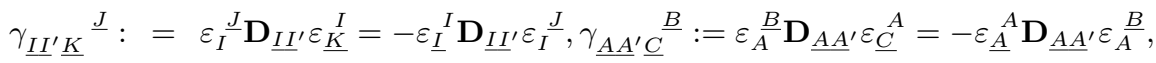

which are equivalent to the spin $\mathrm{d}$-connection from Definition 3.4. This way, we can introduce a canonical and/or Cartan type null-tetradic type calculus etc. For simplicity, we shall omit in the future spinor $h$ - and $v$-index formulas if that will not result in ambiguities or lost of some important properties.

3.2.2. Spinor $\mathbf{d}$-curvature and Bianchi identities. Following a N-adapted $d-$ spinor calculus with abstract indices for nonholonomic splitting $2+2$ (for simplicity, we shall consider "dot" spinor indices; only some examples for Finsler-Cartan configurations will be considered), we prove:

Theorem 3.2. In d-spinor variables,

- the canonical d-commutator (33) (d-torsion (17)) is

$$
\begin{aligned}
\boldsymbol{\Delta}_{\alpha \beta} & =\varepsilon_{\dot{A} \dot{B}} \square_{\dot{A}^{\prime} \dot{B}^{\prime}}+\varepsilon_{\dot{A}^{\prime} \dot{B}^{\prime}} \square_{\dot{A} \dot{B}}, \\
\boldsymbol{\Delta}_{i j} & =\varepsilon_{I J} \square_{I^{\prime} J^{\prime}}+\varepsilon_{I^{\prime} J^{\prime}} \square_{I J},, \boldsymbol{\Delta}_{i a}=\varepsilon_{I A} \square_{I^{\prime} A^{\prime}}+\varepsilon_{I^{\prime} A^{\prime}} \square_{I A}, \ldots
\end{aligned}
$$

where $\square_{\dot{A} \dot{B}}:=\mathbf{D}_{\dot{A}^{\prime}(\dot{A}} \mathbf{D}_{\dot{B})}^{\dot{A}^{\prime}}$ and $\square_{\dot{A}^{\prime} \dot{B}^{\prime}}:=\mathbf{D}_{\dot{A}\left(\dot{A}^{\prime}\right.} \mathbf{D}_{\left.\dot{B}^{\prime}\right)}^{\dot{A}}$ etc;

- the spinor $d$-commutators acting on a $d$-spinor $\varkappa_{\dot{C}}$ result in

$$
\begin{aligned}
\square_{\dot{A} \dot{B}} \varkappa_{\dot{C}} & =\left[\Psi_{\dot{C} \dot{T} \dot{A} \dot{B}}+\Lambda\left(\varepsilon_{\dot{A} \dot{C}} \varepsilon_{\dot{B} \dot{T}}+\varepsilon_{\dot{A} \dot{T}} \varepsilon_{\dot{B} \dot{C}}\right)\right] \varkappa^{\dot{T}}, \\
\square_{\dot{A}^{\prime} \dot{B}^{\prime}} \varkappa_{\dot{C}} & =\Phi_{\dot{C} \dot{T} \dot{A}^{\prime} \dot{B}^{\prime}} \varkappa^{\dot{T}} ;
\end{aligned}
$$

- the Riemann d-tensor (18) is

$$
\begin{aligned}
& \mathbf{R}_{\tau \gamma \alpha \beta}=\Psi_{\dot{T} \dot{C} \dot{A} \dot{B}^{\prime}} \varepsilon_{\dot{A}^{\prime} \dot{B}^{\prime}} \varepsilon_{\dot{T}^{\prime} \dot{C}^{\prime}}+\bar{\Psi}_{\dot{T}^{\prime} \dot{C}^{\prime} \dot{A}^{\prime} \dot{B}^{\prime}} \varepsilon_{\dot{A} \dot{B}} \varepsilon_{\dot{T} \dot{C}}+\Phi_{\dot{T}^{\prime} \dot{C}^{\prime} \dot{A} \dot{B}^{\prime}} \varepsilon_{\dot{A}^{\prime} \dot{B}^{\prime}} \varepsilon_{\dot{T} \dot{C}} \\
& +\bar{\Phi}_{\dot{T} \dot{C} \dot{A}^{\prime} \dot{B}^{\prime}} \varepsilon_{\dot{A} \dot{B}} \varepsilon_{\dot{T}^{\prime} \dot{C}^{\prime}}+2 \Lambda\left(\varepsilon_{\dot{A} \dot{C}} \varepsilon_{\dot{B} \dot{T}^{\prime}} \varepsilon_{\dot{A}^{\prime} \dot{C}^{\prime}} \varepsilon_{\dot{B}^{\prime} \dot{T}^{\prime}}-\varepsilon_{\dot{A} \dot{T}} \varepsilon_{\dot{B} \dot{C}^{\prime}} \varepsilon_{\dot{A}^{\prime} \dot{T}^{\prime}} \varepsilon_{\dot{B}^{\prime} \dot{C}^{\prime}}\right), \\
& \text { for } \Lambda=\bar{\Lambda}=\frac{1}{24}{ }_{s} R ;
\end{aligned}
$$

- the Weyl conformal d-tensor (36) splits into anti-selfdual,

$$
{ }^{-} C_{\alpha \beta \gamma \tau}:=\Psi_{\dot{A} \dot{B} \dot{C} \dot{T}} \varepsilon_{\dot{A}^{\prime} \dot{B}^{\prime}} \varepsilon_{\dot{C}^{\prime} \dot{T}^{\prime}},
$$

and selfdual parts,

$$
{ }^{+} \mathbf{C}_{\alpha \beta \gamma \tau}:=\bar{\Psi}_{\dot{A}^{\prime} \dot{B}^{\prime} \dot{C}^{\prime} \dot{T}^{\prime}} \varepsilon_{\dot{A} \dot{B}} \varepsilon_{\dot{C} \dot{T}},
$$

when $\mathbf{C}_{\alpha \beta \gamma \tau}=-\mathbf{C}_{\alpha \beta \gamma \tau}+{ }^{+} \mathbf{C}_{\alpha \beta \gamma \tau}$.

Similar N-adapted $2+2$ decompositions can be computed for $\tilde{D}_{i}=$ $\tilde{D}_{I I^{\prime}}=\tilde{D}_{I^{\prime} I}, \tilde{D}_{a}=\tilde{D}_{A A^{\prime}}=\tilde{D}_{A^{\prime} A}$. For instance, the components of the Finsler-Cartan curvature (31) can be written

$$
\begin{aligned}
\tilde{R}_{i j k h}= & \tilde{\Psi}_{I J K H} \varepsilon_{I^{\prime} J^{\prime}} \varepsilon_{K^{\prime} H^{\prime}}+\overline{\tilde{\Psi}}_{I^{\prime} J^{\prime} K^{\prime} H^{\prime}} \varepsilon_{I J} \varepsilon_{K H}+\tilde{\Phi}_{I J K^{\prime} H^{\prime}} \varepsilon_{I^{\prime} J^{\prime}} \varepsilon_{K H} \\
& +\tilde{\tilde{\Phi}}_{I^{\prime} J^{\prime} K H_{I J}} \varepsilon_{I J} \varepsilon_{K^{\prime} H^{\prime}}+2 \Lambda\left(\varepsilon_{I K} \varepsilon_{J H} \varepsilon_{I^{\prime} K^{\prime}} \varepsilon_{J^{\prime} H^{\prime}}-\varepsilon_{I H} \varepsilon_{J K} \varepsilon_{I H} \varepsilon_{J K}\right), \\
\tilde{P}_{i j k a}= & \tilde{\Psi}_{I J K A} \varepsilon_{I^{\prime} J^{\prime}} \varepsilon_{K^{\prime} A^{\prime}}+\tilde{\tilde{\Psi}}_{I^{\prime} J^{\prime} K^{\prime} A^{\prime}} \varepsilon_{I J} \varepsilon_{K A}+\tilde{\Phi}_{I J K^{\prime} A^{\prime}} \varepsilon_{I^{\prime} J^{\prime}} \varepsilon_{K A} \\
& +\tilde{\Phi}_{I^{\prime} J^{\prime} K A} \varepsilon_{I J} \varepsilon_{K^{\prime} A^{\prime}}+2 \Lambda\left(\varepsilon_{I K} \varepsilon_{J A} \varepsilon_{I^{\prime} K^{\prime}} \varepsilon_{J^{\prime} A^{\prime}}-\varepsilon_{I A} \varepsilon_{J K} \varepsilon_{I A} \varepsilon_{J K}\right),
\end{aligned}
$$


We note that we can apply the formalism from [1] for any $h$ - and $v$-values (with not "dot" indices) and, in general form for small Greek d-tensor indices, with "dot" indices), if we work in N-adapted frames and keep in mind that Finsler like $\mathrm{d}$-connections $\mathbf{D}_{\alpha}$, or $\tilde{\mathbf{D}}_{\alpha}$, are with torsions completely determined by data $(\mathbf{g}, \mathbf{N})$. Following such rules, we obtain proofs for

Theorem 3.3. - The Bianchi identities (40) transform into

$$
\mathbf{D}_{\dot{B}}^{\dot{A}} \Psi_{\dot{A} \dot{B} \dot{C} \dot{T}}=\mathbf{D}_{(\dot{B}}^{\dot{A}^{\prime}} \Phi_{\dot{C} \dot{T}) \dot{A}^{\prime} \dot{B}^{\prime}}, \mathbf{D}^{\dot{C} \dot{A}^{\prime}} \Phi_{\dot{C} \dot{T} \dot{A}^{\prime} \dot{B}^{\prime}}=-3 \mathbf{D}_{\dot{T} \dot{B}^{\prime}} \Lambda
$$

- the Einstein d-equations (6), (21) and/or (34) transform the first identity into

$\mathbf{D}_{\dot{B}}^{\dot{A}} \Psi_{\dot{A} \dot{B} \dot{C} \dot{T}}=8 \pi G \mathbf{D}_{(\dot{B}}^{\dot{A}^{\prime}} \mathbf{T}_{\dot{C} \dot{T}) \dot{A}^{\prime} \dot{B}^{\prime}}$, see also (35);

- the vacuum field equations for locally anisotropic models with nontrivial cosmological constant $\lambda=6 \Lambda$ are

$$
\Phi_{\dot{C} \dot{T} \dot{A}^{\prime} \dot{B}^{\prime}}=0, \mathbf{D}_{\dot{A} \dot{B}^{\prime}} \Psi_{\dot{A} \dot{B} \dot{C} \dot{T}}=0 ;
$$

- in Cartan-Finsler gravity models with $2+2$ splitting, similar formulas hold for $\mathbf{D} \rightarrow \tilde{\mathbf{D}}$;

- all equations from this theorem transform into similar ones for the LeviCivita connection $\nabla$ if and only if $\boldsymbol{\Delta}_{\alpha \beta}=0$, or $\tilde{\boldsymbol{\Delta}}_{\alpha \beta}=0$, see (33), which is equivalent to (7).

3.2.3. $\mathbf{N}$-adapted conformal transforms. Let us introduce the value

$$
\Upsilon_{\alpha}:=\varpi^{-1} \mathbf{D}_{\alpha} \varpi=\mathbf{D}_{\alpha} \ln \varpi,
$$

where the nonzero positive function $\varpi(u)$ is taken for conformal transforms $\widehat{\mathbf{g}}_{\alpha \beta}:=\varpi^{2} \mathbf{g}_{\alpha \beta}$ (39) from Proposition 2.3. Using the first formula in (41), we conclude that in $\mathrm{N}$-adapted form

$$
\varepsilon_{\dot{A} \dot{B}} \rightarrow \widehat{\varepsilon}_{\dot{A} \dot{B}}=\varpi \varepsilon_{\dot{A} \dot{B}} \text { and } \varepsilon_{\dot{A}^{\prime} \dot{B}^{\prime}} \rightarrow \widehat{\varepsilon}_{\dot{A}^{\prime} \dot{B}^{\prime}}=\varpi \varepsilon_{\dot{A}^{\prime} \dot{B}^{\prime}} .
$$

A corresponding abstract $\mathrm{d}$-spinor calculus for last formula in Theorem 3.2 results in proof of

Proposition 3.1. In $N$-adapted form, $\widehat{\Psi}_{\dot{A} \dot{B} \dot{C} \dot{T}}=\Psi_{\dot{A} \dot{B} \dot{C} \dot{T}}$.

Applying statements of Theorem 3.3, we obtain formulas

$$
\widehat{\mathbf{D}}^{\dot{A} \dot{A}^{\prime}} \Psi_{\dot{A} \dot{B} \dot{C} \dot{T}}=\Upsilon^{\dot{A} \dot{A}^{\prime}} \Psi_{\dot{A} \dot{B} \dot{C} \dot{T}} \text { and } \mathbf{D}_{\dot{A}^{\prime}}^{\dot{T}} \Psi_{\dot{A} \dot{B} \dot{C} \dot{T}}=\mathbf{D}_{(\dot{B}}^{\dot{B}^{\prime}} \mathbf{P}_{\dot{A}) \dot{C} \dot{A}^{\prime} \dot{B}^{\prime}}
$$

and $\widehat{\mathbf{D}}_{(\dot{B}}^{\dot{B}^{\prime}} \widehat{\boldsymbol{\Phi}}_{\dot{C} \dot{T}) \dot{A}^{\prime} \dot{B}^{\prime}}=\Upsilon_{\dot{A}^{\prime}}^{\dot{A}} \Psi_{\dot{A} \dot{B} \dot{C} \dot{T}}$

Remark 3.1. Vacuum solutions of Einstein equations in general relativity and models of Finsler-Cartan gravity with $\mathbf{D}$, or $\tilde{\mathbf{D}}$, are not conformally invariant. This follows from the fact that even $\boldsymbol{\Phi}_{\dot{C} \dot{T} \dot{A}^{\prime} \dot{B}^{\prime}}=0$ the above formula with $\widehat{\mathbf{D}}_{(\dot{B}}^{\dot{B}^{\prime}} \widehat{\boldsymbol{\Phi}}_{\dot{C} \dot{T}) \dot{A}^{\prime} \dot{B}^{\prime}}$ does not result in zero $\widehat{\boldsymbol{\Phi}}_{\dot{C} \dot{T} \dot{A}^{\prime} \dot{B}^{\prime}}$. Nevertheless, it should be emphasized here that such results are for a fixed $N$-connection structure $\mathbf{N}$, or $\tilde{\mathbf{N}}$. We can transform some data $(\mathbf{g}, \mathbf{N})$ with (non) zero $\boldsymbol{\Phi}_{\dot{C} \dot{T} \dot{A}^{\prime} \dot{B}^{\prime}}$ into certain $\left({ }^{\eta} \mathbf{g},{ }^{\eta} \mathbf{N}\right)$ with, for instance, ${ }^{\eta} \boldsymbol{\Phi}_{\dot{C} \dot{T} \dot{A}^{\prime} \dot{B}^{\prime}}=0$, and/or 
$\eta \widehat{\mathbf{D}}_{(\dot{B}}^{\dot{B}^{\prime}} \eta \widehat{\boldsymbol{\Phi}}_{\dot{C} \dot{T}) \dot{A}^{\prime} \dot{B}^{\prime}}=\eta \Upsilon_{\dot{A}^{\prime}}^{\dot{A}} \eta \Psi_{\dot{A} \dot{B} \dot{C} \dot{T}}=0$, for instance, following the anholonomic deformation method $[5,14,19]$.

Via $\mathrm{N}$-adapted $\mathrm{d}-$-spinor calculus, we can prove

Theorem 3.4. Under $N$-adapted conformal transforms, the values determining the vacuum Einstein equations for $\mathbf{D}$ transform following rules

$$
\begin{aligned}
\widehat{\boldsymbol{\Phi}}_{\dot{C} \dot{T} \dot{A}^{\prime} \dot{B}^{\prime}} & =\boldsymbol{\Phi}_{\dot{C} \dot{T} \dot{A}^{\prime} \dot{B}^{\prime}}=-\mathbf{D}_{\dot{C}\left(\dot{B}^{\prime}\right.} \Upsilon_{\left.\dot{A}^{\prime}\right) \dot{T}}+\Upsilon_{\dot{C}\left(\dot{B}^{\prime}\right.} \Upsilon_{\left.\dot{A}^{\prime}\right) \dot{T}} \\
& =\varpi \mathbf{D}_{\dot{C}\left(\dot{B}^{\prime}\right.} \mathbf{D}_{\left.\dot{A}^{\prime}\right) \dot{T}} \varpi^{-1}=-\varpi^{-1} \widehat{\mathbf{D}}_{\dot{C}\left(\dot{B}^{\prime}\right.} \widehat{\mathbf{D}}_{\left.\dot{A}^{\prime}\right) \dot{T}} \varpi \\
4 \varpi^{2} \widehat{\Lambda} & =4 \Lambda+\left(\mathbf{D}^{\alpha} \Upsilon_{\alpha}+\Upsilon^{\alpha} \Upsilon_{\alpha}\right)=4 \Lambda+\varpi^{-1} \square \varpi
\end{aligned}
$$

for $\square:=\mathbf{D}^{\alpha} \mathbf{D}_{\alpha}$.

Using the operators $\mathbf{D}_{\dot{C} \dot{B}^{\prime}}$ and/or $\tilde{\mathbf{D}}_{\dot{C} \dot{B}^{\prime}}$, we can construct other conformally $\mathrm{N}$-adapted invariant values, for instance, a Finsler like Bach d-tensor (it can be constructed similarly to formulas (6.8.42)-(6.8.45) in [1] but for d-connections).

Finally, we note that we can formulate spinor N-adapted differential geometries and derive following geometric/N-adapted variational principles certain gravitational and matter field equations for $\mathbf{V}, \operatorname{dim} \mathbf{V}=n+m ; n, m \geq$ 2 , generalizing for higher dimensions the tensor and spinor abstract index formalism.

\section{Nonholonomic (Finsler) Twistors}

The twistor theory was elaborated with a very general goal to translate the standard physics in the language of complex manifolds mathematics when spacetime points and fundamental properties and field interactions are derived from certain fundamental principles being generalized former constructions for spinor algebra and geometry. The approach is characterized by certain important results in generating exact solutions of fundamental matter field equations, twistor methods of quantization, formulating conservation laws in gravity and encoding, for instance, of (anti) self-dual Yang-Mills and gravitational interactions, see details in Refs. [1, 2, 3, 4].

In some sense, our research interests are twofold: 1) The first aim to understand to what extend the Twistor Program can be generalized for modified Finsler type spacetime geometries. 2) Nonholonomic (Finsler) methods happen to be very effective in elaborating new geometric methods of constructing exact solutions and quantization of gravity theories. The second aim is to clarify how such approaches can be related to spinor and twistor geometry? Our constructions should provide not only "pure" academic generalizations of twistor geometry for "more sophisticate" spacetime models with local anisotropies. Finsler like variables can be introduced even in general relativity (similarly to various former tetradic, spinor etc approaches) which give us new possibilities for developing the twistor theory for curved spaces and generic off-diagonal gravitational and matter field interactions. 
The aim of this section is to define twistors for nonholonomic (Finsler) spaces and show how such constructions can be globalized on curved spaces via nonholonomic deformations of fundamental geometric structures.

\subsection{Twistor equations for nonholonomic $2+2$ splitting}

Originally, twistors were introduced for complexified projective models of flat Minkowski spacetimes using the two-spinor formalism. For nonholonomic manifolds enabled with $\mathrm{N}$-connection structure, we can consider analogs of flat spaces determined by data $(\mathbf{g}, \mathbf{N}, \mathbf{D})$ for which the N-adapted Riemannian curvature and the conformal Weyl d-tensors are zero (see Theorem 3.2). In general, such geometries are curved ones because the curvature of $\nabla$ is not zero. The spinor constructions are similar to those for (pseudo) Euclidean spaces if there are used N-adapted frames (8) and (9).

4.1.1. Definition of nonholonomic twistors. Let us consider analogs of flat twistors on spaces enabled with $\mathrm{N}$-connection structure.

Definition 4.1. The nonholonomic twistor equations are

$$
\mathbf{D}_{\dot{A}^{\prime}}^{(\dot{A}} \omega^{\dot{B})}=0
$$

Let us formulate the conditions when such equations are conformally invariant in N-adapted form. Choosing $\widehat{\omega}^{\dot{B}}=\omega^{\dot{B}}$, we can compute

$$
\widehat{\mathbf{D}}_{\dot{A} \dot{A^{\prime}}}, \widehat{\omega}^{\dot{B}}=\widehat{\mathbf{D}}_{\dot{A} \dot{\prime}}, \widehat{\omega}^{\dot{B}}+\delta_{\dot{A}}^{\dot{B}} \Upsilon_{\dot{C} \dot{A}} \text {, and } \widehat{\mathbf{D}}_{\dot{A}^{\prime}}^{(\dot{A}} \widehat{\omega}^{\dot{B})}=\varpi^{-1} \mathbf{D}_{\dot{A}^{\prime}}^{(\dot{A}} \omega^{\dot{B})}
$$

where $\Upsilon_{\dot{C} \dot{A}^{\prime}}$ is given by the conformal d-vector (45). ${ }^{10}$

Lemma 4.1. The nonholonomic twistor equations (46) are compatible if and only if

$$
\Psi_{\dot{T} \dot{A} \dot{B} \dot{C}} \omega^{\dot{T}}=0 .
$$

Proof. It follows from $\mathbf{D}^{\dot{A}^{\prime}(\dot{C}} \mathbf{D}_{\dot{A}^{\prime}}^{\dot{A}} \omega^{\dot{B})}=-\square^{(\dot{C} \dot{A}} \omega^{\dot{B})}=-\Psi_{\dot{T}}^{\dot{A} \dot{B}} \dot{C} \omega^{\dot{T}}$.

Using this lemma and via straightforward verifications in $\mathrm{N}$-adapted frames, we can prove

Theorem 4.1. If the compatibility conditions (48) are satisfied, we can solve the nonholonomic twistor equations (46) in general form,

$$
\begin{aligned}
\omega^{\dot{B}} & =\stackrel{\circ}{\omega}^{\dot{B}}-i u^{\dot{B} \dot{B}^{\prime \prime}}{\stackrel{\pi}{\pi^{\prime}}} \text { and } \pi_{\dot{B} \prime}=\stackrel{\circ}{\pi}_{\dot{B} \prime}, \\
\mathbf{D}_{\dot{B} \dot{B} \prime}{ }^{\dot{C}} \omega^{\dot{C}} & =-i \delta_{\dot{B}}^{\dot{C}} \pi_{\dot{B} \prime},
\end{aligned}
$$

where the point $u^{\dot{B} \dot{B}^{\prime}} \in \mathbf{V}, i^{2}=-1$, and $\stackrel{\circ}{\omega} \dot{B}$ and $\stackrel{\circ}{\pi}_{\dot{B} \text {, }}$ are constant values with respect to $N$-adapted frames (8) and (9) for which $\Psi_{\dot{A} \dot{B} \dot{C} \dot{T}}=0$.

We can generalize the concept of twistors for flat spaces to nonholonomic manifolds which are conformally flat in $\mathrm{N}$-adapted form:

\footnotetext{
${ }^{10}$ For simplicity, we shall consider that the spinor $\omega^{\dot{B}}$ does not posses an electromagnetic charge.
} 
Definition 4.2. An nonholonomic (equivalently, anholonomic) twisor space $\mathbb{T}^{\dot{\alpha}}$ is a four dimensional complex vector space (with real eight dimensions) determined by elements of type $\boldsymbol{Z}^{\dot{\alpha}}=\left(\omega^{\dot{A}}, \pi_{\dot{A}^{\prime}}\right)$ with the two spinor components $\boldsymbol{Z}^{\dot{A}}=\omega^{\dot{A}}$ and $\boldsymbol{Z}_{\dot{A}^{\prime}}=\pi_{\dot{A}^{\prime}}$ defined by solutions of type (49).

Doted indices are used in order to emphasize that we work in $\mathrm{N}$-adapted form. This allows us to preserve with respect to $\mathrm{N}$-adapted frames certain similarity to formulas from [1]. If $\mathbf{D} \rightarrow \nabla$, for conformally flat spaces, we obtain $\boldsymbol{Z}^{\dot{\alpha}} \rightarrow Z^{\alpha} \in \mathbb{T}^{\alpha}$, i.e. standard Penrose's twistors. Such constructions and relations depends on a point ${ }^{0} u$ fixing a coordinate system.

Remark 4.1. In a similar form, we can introduce the space of nonholonomic dual twistors $\mathbb{T}_{\dot{\alpha}}$ with elements $\boldsymbol{W}_{\dot{\alpha}}=\left(\boldsymbol{W}_{\dot{A}}=\lambda_{\dot{A}}, \boldsymbol{W}^{\dot{A}^{\prime}}=\mu^{\dot{A}^{\prime}}\right)$, where

$$
\begin{aligned}
\lambda_{\dot{A}} & =\dot{\lambda}_{\dot{A}} \text { and } \mu^{\dot{A}^{\prime}}=\stackrel{\circ}{\mu}^{\dot{A}^{\prime}}+i u{ }^{\dot{A} \dot{A}^{\prime}} \dot{ }_{\dot{A}}, \\
\mathbf{D}_{\dot{A} \dot{A}^{\prime}} \mu^{\dot{B} \prime} & =i \delta_{\dot{A}^{\prime}}^{\dot{B}^{\prime}} \lambda_{\dot{A}},
\end{aligned}
$$

are solutions of the dual nonholonomic twistor equations $\mathbf{D}_{\dot{A}}^{\left(\dot{A}^{\prime}\right.} \mu^{\left.\dot{B}^{\prime}\right)}=0$.

The complex conjugation of nonholonomic (dual) twistors follows the rules

$$
\overline{\boldsymbol{Z}^{\dot{\alpha}}}=\overline{\boldsymbol{Z}}_{\dot{\alpha}}:=\left(\bar{\pi}_{\dot{A}}, \bar{\omega}^{\dot{A}^{\prime}}\right) \text { and } \overline{\boldsymbol{W}_{\dot{\alpha}}}=\overline{\boldsymbol{W}}^{\dot{\alpha}}:=\left(\bar{\mu}^{\dot{A}}, \bar{\lambda}_{\dot{A}^{\prime}}\right) \text {. }
$$

We can consider higher valence twistors, for instance, $\mathbf{X}_{\dot{\beta}}^{\dot{\alpha}}$ where N-adapted twistor indices transform respectively following rules (49) and (50) taken "-" or "+" before complex unity $i$.

4.1.2. Geometric/physical meaning of anholonomic twistors. A nonholonomic frame structure prescribes a corresponding spiral configuration for twistors and their conformal transforms.

Definition and Corollary 4.3. The class of curved spaces generated by anholonomy relations (12) subjected to the compatibility conditions (48) is characterized by anholonomic spirality

$$
\dot{\mathbf{s}}:=\frac{1}{2} \boldsymbol{Z}^{\dot{\alpha}} \overline{\boldsymbol{Z}}_{\dot{\alpha}}
$$

which is invariant under $N$-adapted conformal transforms.

Proof. It follows from verification that $\boldsymbol{Z}^{\dot{\alpha}} \overline{\boldsymbol{Z}}_{\dot{\alpha}}=\widehat{\boldsymbol{Z}}^{\dot{\alpha}} \widehat{\boldsymbol{Z}}_{\dot{\alpha}}$ (using formulas (47) and (49)).

In both holonomic and nonholonomic cases, the simplest geometric interpretation is possible for the so-called isotropic twistors when $\boldsymbol{Z}^{\dot{\alpha}} \overline{\boldsymbol{Z}}_{\dot{\alpha}}=0$. Fixing a value ${\stackrel{\circ}{\dot{B}_{\prime}}} \neq 0$, we get from (49) that

$$
u^{\dot{B} \dot{B} \prime}=\stackrel{\dot{\omega}}{\dot{B}} \overline{\dot{\omega}}^{\dot{B}^{\prime}} / i \overline{\dot{\omega}}^{\dot{A}^{\prime}}{\stackrel{\circ}{\pi_{A^{\prime}}}}+\tau \bar{\pi}^{\dot{B}} \pi^{\dot{B} \prime}, \tau \in \mathbb{R},
$$

describes a light ray propagating in $\mathrm{N}$-adapted form in an effective locally anisotropic media and/or a curved spacetime with geometric objects induced 
by nontrivial anholonomy coefficients . If ${\stackrel{\circ}{\dot{B}^{\prime}}}=0$, such a light ray is moved to infinity.

We can also characterize massless particles with momentum, rotation and spirality propagating in effective curved spaces derived for certain anholonomy relations of moving frames. Taking $Z^{\dot{\alpha}}=\left(\omega^{\dot{A}}, \pi_{\dot{A}^{\prime}}\right)$ with $\pi_{\dot{A}^{\prime}} \neq 0$, we construct

$$
p_{\dot{A}^{\prime} \dot{A}^{\prime}}:=\bar{\pi}_{\dot{A}} \pi_{\dot{A}^{\prime}}, M^{\dot{A} \dot{A}^{\prime} \dot{B} \dot{B}^{\prime}}:=i \omega^{(\dot{A}} \bar{\pi}^{\dot{B})} \varepsilon^{\dot{A}^{\prime} \dot{B} \prime}-i \bar{\omega}^{\left(\dot{A}^{\prime}\right.} \pi^{\left.\dot{B}^{\prime}\right)} \varepsilon^{\dot{A} \dot{B}}
$$

and spin d-vector $S_{\alpha}=\frac{1}{2} e_{\alpha \beta \gamma \tau} p^{\beta} M^{\gamma \tau}=\dot{\mathbf{s}} p_{\alpha}$, where $e_{\alpha \beta \gamma \tau}$ is the absolute antisymmetric $\mathrm{d}$-tensor and $\dot{\mathbf{s}}$ is computed as in (51). In local $\mathrm{N}$-adapted form, such a physical interpretation of nonholonomic twistors is possible with respect to bases of type (8) and (9) for which $\Psi_{\dot{A} \dot{B} \dot{C} \dot{T}}=0$. This describes a massless particle moving in a subclass of curved spaces with nontrivial curvature for $\nabla$ when certain anholonomic constraints are imposed.

\subsection{Finsler twistors on tangent bundles}

Originally, the Finsler-Cartan geometry was constructed on tangent bundles with $\boldsymbol{D}=\tilde{\boldsymbol{D}}$ and $4+4$ splitting as we explained in section 2.2.3. The corresponding Weyl d-tensor $\tilde{\mathbf{C}}_{\tau \alpha \beta \gamma}$ is computed using formulas (36) but for curvature coefficients (31) and curvature spinors (44).

Definition 4.4. The twistor equations for Finsler-Cartan geometries are

$$
\mathbf{D}_{I^{\prime}}^{(I} \omega^{J)}=0, \mathbf{D}_{A^{\prime}}^{(A} \omega^{B)}=0 .
$$

Choosing $\widehat{\omega}^{J}=\omega^{J}, \widehat{\omega}^{B}=\omega^{B}$, we can compute

$$
\begin{aligned}
\widehat{\mathbf{D}}_{I I^{\prime}} \widehat{\omega}^{J} & =\widehat{\mathbf{D}}_{I I^{\prime}} \widehat{\omega}^{J}+\delta_{I}^{J} \tilde{\Upsilon}_{K J^{\prime}} \text { and } \widehat{\mathbf{D}}_{I^{\prime}}^{\left(I \widehat{\omega}^{J}\right)}=\varpi^{-1} \mathbf{D}_{I^{\prime}}^{(I} \omega^{J)}, \\
\widehat{\mathbf{D}}_{A A^{\prime}} \widehat{\omega}^{B} & =\widehat{\mathbf{D}}_{A A^{\prime}} \widehat{\omega}^{B}+\delta_{A}^{B} \tilde{\Upsilon}_{C A^{\prime}} \text { and } \widehat{\mathbf{D}}_{A^{\prime}}^{\left(A \widehat{\omega}^{B)}\right.}=\varpi^{-1} \mathbf{D}_{A^{\prime}}^{(A} \omega^{B)^{\prime} \prime},
\end{aligned}
$$

where $\tilde{\Upsilon}_{I I^{\prime}}:=\tilde{\mathbf{D}}_{I I^{\prime}} \ln \varpi \Upsilon_{\dot{C} \dot{A}^{\prime}}, \tilde{\Upsilon}_{A A^{\prime}}:=\tilde{\mathbf{D}}_{A A^{\prime}} \ln \varpi$ are constructed similarly to the conformal d-vector (45).

If $\boldsymbol{D}=\tilde{\boldsymbol{D}}$, we can obtain from the Theorem 3.2 the

Corollary 4.1. The anti-sefldual Weyl $d$-spinors corresponding to the Cartan $d$-curvature $\tilde{\mathbf{R}}_{\beta \gamma \tau}^{\alpha}=\left\{\tilde{R}_{h j k}^{i}, \tilde{P}_{j k a}^{i}, \tilde{S}_{b c d}^{a}\right\}$ (31) are characterized by $h$ - and $v$-components $\left\{\tilde{\Psi}_{L I J K}, \tilde{\Psi}_{D I J K}, \tilde{\Psi}_{D A B C}\right\}$.

This results in a set of three conditions of compatibility:

Lemma 4.2. The Finsler-Cartan twistor equations (46) are compatible if and only if

$$
\tilde{\Psi}_{L I J K} \omega^{L}=0, \tilde{\Psi}_{D I J K} \omega^{D}=0, \tilde{\Psi}_{D A B C} \omega^{D}=0 .
$$

All results on Finsler-Cartan twistors can be proved using formal Sasaki lifts $\mathbf{g} \rightarrow \tilde{\mathbf{g}}$ (28) and $\mathbf{N} \rightarrow \tilde{\mathbf{N}}$ (27) with spinor coefficients (41) for the constructions with nonholonomic twistors and canonical $\mathrm{d}-$-connections. 
Theorem 4.2. If the compatibility conditions (53) are satisfied, we can solve the nonholonomic twistor equations (52) in general form for $h$-components,

$$
\begin{aligned}
\omega^{J} & =\stackrel{\leftrightarrow}{\omega}^{J}-i u{\stackrel{J J \prime}{\pi_{J \prime}}} \text { and } \pi_{J \prime}=\stackrel{\circ}{\pi}_{J \prime}, \\
\mathbf{D}_{J J} \omega^{K} & =-i \delta_{J}^{K} \pi_{J \prime},
\end{aligned}
$$

and for $v$-components

$$
\begin{aligned}
\omega^{B} & =\stackrel{\omega}{\omega}^{B}-i u{ }^{B{ }^{\prime \prime}} \stackrel{\circ}{\pi}_{B \prime} \text { and } \pi_{B \prime}=\stackrel{\circ}{\pi \prime}_{B \prime}, \\
\mathbf{D}_{B B \prime} \omega^{C} & =-i \delta_{B}^{C} \pi_{B \prime},
\end{aligned}
$$

where the point $u^{\alpha}=\left(u^{I{ }^{\prime \prime}}, u^{A{ }^{A \prime}}\right) \in \mathbf{T M}$ and constant values are considered with respect to $N$-adapted frames (8) and (9) when the conditions (53) are satisfied.

We can generalize the concept of twistors for flat spaces to nonholonomic manifolds which are conformally flat in $\mathrm{N}$-adapted form:

Definition 4.5. $\quad$ - A horizontal twisor space $h \mathbb{T}^{\dot{\alpha}}$ is a four dimensional complex vector space (with real eight dimensions) determined by elements of type $h \boldsymbol{Z}^{i}=\left(\omega^{I}, \pi_{I^{\prime}}\right), \dot{i}=1,2,3,4$ with the two spinor components $h \boldsymbol{Z}^{I}=\omega^{I}$ and $\boldsymbol{Z}_{I^{\prime}}=\pi_{I^{\prime}}$ defined by solutions of type (54).

- A vertical twisor space $v \mathbb{T}^{\dot{\alpha}}$ is a four dimensional complex vector space (with real eight dimensions) determined by elements of type $h \boldsymbol{Z}^{\dot{a}}=$ $\left(\omega^{A}, \pi_{A^{\prime}}\right), \dot{a}=5,6,7,8$ with the two spinor components $v \boldsymbol{Z}^{A}=\omega^{A}$ and $\boldsymbol{Z}_{A^{\prime}}=\pi_{A^{\prime}}$ defined by solutions of type (55).

We conclude that nonholonomic twistor constructions for the FinslerCartan spaces dub as $h$ - and $v$-components the values introduced via canonical $\mathrm{d}$-connections on $\mathbf{V}$. Re-defining the abstract index formalism for $\mathrm{d}-$ tensors and $\mathrm{d}$-spinors, all formulas can be proved by similarity in $\mathrm{N}$-adapted frames.

\subsection{Nonholonomic local and global twistors}

On N-adapted conformally flat nonholonomic manifolds, the solutions (49) of generalized twistor equations (46) define certain global anholonomic twistor structures. If the conditions (53) are not satisfied, we can only define a nonholonomic twistor bundle on a $\boldsymbol{V}$ when the geometric object depend on base manifold points. This does not define an alternative description of nonholonomic manifolds (and Finsler-Cartan geometries) in terms of certain generalized nonholonomic twistor spaces. For a prescribed $\mathrm{N}$-connection structure $N$, we can construct $\mathrm{N}$-adapted local twistors with properties similar to those of holonomic twistors considered in Chapter 6, paragraph 9, in [1].

Nevertheless, nonholonomic/ Finsler spaces are characterized by more rich geometric structures which provide us new possibilities and methods for constructing new classes of generalized twistor - Finsler spaces and applications in general relativity and modifications. We study two models of $\mathrm{N}$-adapted twistor spaces in local and global forms. 
4.3.1. $\mathbf{N}$-adapted local twistors and torsionless conditions. Let us consider a point $u \in \mathbf{V}$ for nonholonomic data $(\mathbf{g}, \mathbf{N}, \mathbf{D})$.

Definition 4.6. A local $N$-adapted twistor ${ }_{u} \mathbf{Z}^{\dot{\alpha}}$ (in brief, local d-twistor) in a point $u$ is given by a couple of $N$-adapted two-spinors $\left({ }_{u} \omega^{\dot{A}},{ }_{u} \pi_{\dot{A}^{\prime}}\right)$ in this point, which in a chosen anholonomic frame (8) and (9) satisfied the rules: if $\mathbf{g}_{\alpha \beta} \rightarrow \widehat{\mathbf{g}}_{\alpha \beta}:=\varpi^{2} \mathbf{g}_{\alpha \beta}$ then

$$
{ }_{u} \mathbf{Z}^{\dot{\alpha}}=\left({ }_{u} \omega^{\dot{A}}, \pi_{\dot{A}^{\prime}}\right) \rightarrow{ }_{u} \widehat{\mathbf{Z}}^{\dot{\alpha}}=\left({ }_{u} \widehat{\omega}^{\dot{A}}={ }_{u} \omega^{\dot{A}},{ }_{u} \widehat{\pi}_{\dot{A}^{\prime}}={ }_{u} \pi_{\dot{A}^{\prime}}+i \Upsilon_{\dot{A} \dot{A}^{\prime}}{ }_{u} \omega^{\dot{A}}\right) .
$$

The local d-vectors ${ }_{u} \mathbf{Z}^{\dot{\alpha}}$ and ${ }_{u} \widehat{\mathbf{Z}}^{\dot{\alpha}}$ depend functionally, respectively, on $(\mathbf{g}, \mathbf{N}, \mathbf{D}, \varpi)$. The set $\mathbf{Z}^{\dot{\alpha}}=\cup_{u \in \mathbf{V}}\left({ }_{u} \mathbf{Z}^{\dot{\alpha}}\right)$ of all local twistors ${ }_{u} \mathbf{Z}^{\dot{\alpha}}$ taken in all points $u$ of $\mathbf{V}$ defines a vector bundle, when the fiber in $u$ is a complex four dimensional vector space (i. e. the spaces of local $\mathrm{N}$-adapted twistors in $u$ ). Such a vector bundle is nonholonomic being endowed with $\mathrm{N}$-connection structure. For simplicity, we shall omit the left low label "u" and write a local twistor as $\mathbf{Z}^{\dot{\alpha}}$ if that will not result in ambiguities.

In $\mathrm{N}$-adapted (and/or general local) form, the connection $\nabla$ can be constructed to possess zero coefficients in a point and/or along a curve though such a point (the so-called normal coordinates). This allows us to define transports of usual local twistors along curves with tangent vector fields $t^{\dot{A} \dot{A}^{\prime}} \subset T \mathbf{V}$. We can generalize such formulas for $\mathrm{d}$-connections $\mathbf{D}$ and $\mathrm{d}-$ vectors $\mathbf{t}^{\dot{A} \dot{A}^{\prime}}$ and consider a local d-twistor $\mathbf{Z}^{\dot{\alpha}}$ which is constant in $\mathrm{N}$-adapted direction $\mathbf{t}^{\alpha}$, when

$$
\begin{aligned}
t^{\dot{A} \dot{A}^{\prime}} \mathbf{D}_{\dot{A} \dot{A}^{\prime}} \omega^{\dot{B}}+i t^{\dot{B} \dot{A}^{\prime}} \pi_{\dot{A}^{\prime}} & =0, \\
t^{\dot{A} \dot{A}^{\prime}} \mathbf{D}_{\dot{A} \dot{A}^{\prime}} \pi_{\dot{B}^{\prime}}+i t^{\dot{A} \dot{A}^{\prime}} \mathbf{P}_{\dot{A} \dot{A}^{\prime} \dot{B} \dot{B}^{\prime}} \omega^{\dot{B}} & =0,
\end{aligned}
$$

where $\mathbf{P}_{\dot{A} \dot{A}^{\prime} \dot{B} \dot{B}^{\prime}}$ is related to the Ricci d-tensor and scalar curvature of $\mathbf{D}$ as in formula (37) and a curve is defined in vicinity of a point ${ }^{0} u$ in the form $u^{\dot{A} \dot{A}^{\prime}}(\tau)={ }^{0} u^{\dot{A} \dot{A}^{\prime}}+t^{\dot{A} \dot{A}^{\prime}} \tau$, for a real parameter $\tau$. The d-twistor transport equations (56) have constant twistor solutions in ${ }^{0} u$ and along $u(\tau)$ which in any point satisfy the conditions

$$
\mathbf{D}_{\dot{A}^{\prime}}^{(\dot{A}} \omega^{\dot{B})}=0 \text { and } \pi_{\dot{A}^{\prime}}=\frac{1}{2} i \mathbf{D}_{\dot{A} \dot{A}^{\prime}} \omega^{\dot{A}} .
$$

Corollary 4.2. The solutions for local d-twistors (57) can be globalized on $\mathbf{V}$ if and only if the conditions (48) are satisfied, for instance, if the Weyl d-spinor vanishes.

Definition 4.7. The $N$-adapted covariant derivative operator (d-connection) along $\mathbf{t}^{\alpha}$ in the space of local d-twistors is by definition

$$
{ }_{\mathbf{t}} \mathbf{D}:=t^{\dot{A} \dot{A}^{\prime}} \mathbf{D}_{\dot{A} \dot{A}^{\prime}}
$$

The local d-twistor $\mathrm{d}$-connection (58) allows us to compute the variation of $\mathbf{Z}^{\dot{\alpha}}$ along $u(\tau)$, following formulas (56) with nonzero right sides,

$$
{ }_{\mathbf{t}} \mathbf{D} \mathbf{Z}^{\dot{\alpha}}=\left(t^{\dot{A} \dot{A}^{\prime}} \mathbf{D}_{\dot{A} \dot{A}^{\prime}} \omega^{\dot{B}}+i t^{\dot{B} \dot{A}^{\prime}} \pi_{\dot{A}^{\prime}}, t^{\dot{A} \dot{A}^{\prime}} \mathbf{D}_{\dot{A} \dot{A}^{\prime}} \pi_{\dot{B}^{\prime}}+i t^{\dot{A} \dot{A}^{\prime}} \mathbf{P}_{\dot{A} \dot{A}^{\prime} \dot{B} \dot{B}^{\prime}} \omega^{\dot{B}}\right) .
$$


Here we note that in similar form we can define dual local $\mathrm{d}$-twistors of type $\boldsymbol{W}_{\dot{\alpha}}=\left(\lambda_{\dot{A}}, \mu^{\dot{A}^{\prime}}\right)$ with $\mathrm{N}$-adapted conformally invariant scalar product

$$
\boldsymbol{W}_{\dot{\alpha}} \mathbf{Z}^{\dot{\alpha}}:=\lambda_{\dot{A}} \omega^{\dot{A}}+\mu^{\dot{A}^{\prime}} \pi_{\dot{A}^{\prime}}
$$

and property that

$$
{ }_{\mathbf{t}} \mathbf{D}\left(\boldsymbol{W}_{\dot{\alpha}} \mathbf{Z}^{\dot{\alpha}}\right)=\left({ }_{\mathbf{t}} \mathbf{D} \boldsymbol{W}_{\dot{\alpha}}\right) \mathbf{Z}^{\dot{\alpha}}+\boldsymbol{W}_{\dot{\alpha}}\left({ }_{\mathbf{t}} \mathbf{D} \mathbf{Z}^{\dot{\alpha}}\right) .
$$

Definition and Lemma 4.8. The curvature $d$-tensor of local $d$-twistor $d$-connection is

$$
i\left({ }_{\mathbf{t}} \mathbf{D}{ }_{\mathbf{v}} \mathbf{D}-{ }_{\mathbf{v}} \mathbf{D}{ }_{\mathbf{t}} \mathbf{D}-{ }_{[\mathbf{t}, \mathbf{v}]} \mathbf{D}\right) \mathbf{Z}^{\dot{\beta}}=\mathbf{Z}^{\dot{\alpha}} \mathbf{K}_{\dot{\alpha}}^{\dot{\beta}}(\mathbf{t}, \mathbf{v})=\mathbf{t}^{\mu} \mathbf{v}^{\nu} \mathbf{K}_{\dot{\alpha} \mu \nu}^{\dot{\beta}},
$$

for two d-vectors $\mathbf{t}^{\mu}=\mathbf{t}^{\dot{M} \dot{M}^{\prime}}, \mathbf{v}^{\nu}=\mathbf{v}^{\dot{N} \dot{N}^{\prime}} \in T \mathbf{V}$ and computed with $N_{-}$ adapted coefficients,

$$
\mathbf{K}_{\dot{\alpha} \mu \nu}^{\dot{\beta}}=\left(\begin{array}{cc}
i \varepsilon_{\dot{M}^{\prime} \dot{N}^{\prime}} \boldsymbol{\Psi}_{\dot{A} \dot{M} \dot{N}}^{\dot{B}} & \varepsilon_{\dot{M} \dot{N}} \mathbf{D}_{\dot{A}}^{\dot{A}^{\prime}} \overline{\boldsymbol{\Psi}}_{\dot{A}^{\prime} \dot{M}^{\prime} \dot{N}^{\prime}}^{\dot{B}^{\prime}}+\varepsilon_{\dot{M}^{\prime} \dot{N}^{\prime}} \mathbf{D}_{\dot{B^{\prime}}}^{\dot{B}} \boldsymbol{\Psi}_{\dot{A} \dot{M} \dot{N}}^{\dot{B}} \\
0 & -i \varepsilon_{\dot{M} \dot{N}} \overline{\boldsymbol{\Psi}}_{\dot{A}^{\prime} \dot{M}^{\prime} \dot{N}^{\prime}}
\end{array}\right) .
$$

Above constructions are determined by data $(\mathbf{g}, \mathbf{N}, \mathbf{D}=\nabla+\mathbf{Q})$, see (5). In general, they can be redefined for data $(\mathbf{g}, \nabla)$ using nonholonomic deformations.

Theorem 4.3. We can globalize in nonholonomic form the local twistor constructions for $\nabla$ if there is a $N$-connection structure $\mathbf{N}$ and associated $\mathbf{D}$ for which $\mathbf{K}_{\dot{\alpha} \mu \nu}^{\dot{\beta}}=0$ and $\boldsymbol{\Delta}_{\alpha \beta}=0$.

Proof. It is a consequence of conditions of Theorem 3.2 when $\boldsymbol{\Delta}_{\alpha \beta}=0$ are equivalent to (7), i.e. the nonholonomically induced torsion (by $(\mathbf{g}, \mathbf{N})$ ) became zero. This is compatible with Conclusion 2.2 when the conformal Weyl d-tensor/d-spinor for $\mathbf{D}$ can be zero but similar values for $\nabla$ are not trivial. Such linear connections are different even in some $\mathrm{N}$-adapted frames they can be characterized by the same set of coefficients (transformation laws under frame/coordinate changing are different).

We conclude that via nonholonomic transforms we can generate some compatible global nonholonomic twistor equations even the standard twistor equations are not compatible for general curved spacetimes.

4.3.2. Global extensions of $\mathbf{N}$-adapted twistor structures. Our idea is to play with such nonholonomic distributions $N$ which allows us to define spinors and twistors in very general forms.

Claim 4.1. For any (pseudo) Riemannian metric structure $\mathbf{g}$ on a manifold $V$ (or a fundamental Finsler function on tangent bundle TM), we can prescribe a $N$-connection which allows us to globalize $N$-adapted local twistor structures. 
Proof. Let us fix a d-metric (13) with coefficients $\mathbf{g}_{\alpha^{\prime} \beta^{\prime}}:=\varpi^{2}(u) \eta_{\alpha \beta}$ with $\eta_{\alpha \beta}$ being diagonal constants of any necessary signature $( \pm 1, \pm, \ldots, \pm)$, with respect to some $\mathbf{e}^{\beta^{\prime}}=\left(e^{j^{\prime}}=d x^{j^{\prime}}, \mathbf{e}^{b^{\prime}}=d y^{b^{\prime}}+N_{i^{\prime}}^{b^{\prime}} d x^{i^{\prime}}\right)$. For such a $\mathrm{d}$-metric and $\mathrm{N}$-adapted co-bases, we can verify that $\mathbf{C}_{\tau^{\prime} \alpha^{\prime} \beta^{\prime} \gamma^{\prime}}=0$ as a consequence of Proposition 2.3. We can redefine data ( (13), $\left.\mathbf{g}_{\alpha^{\prime} \beta^{\prime}}\right)$ in a coordinate form (14) with coefficients $(15)$ (with primed indices, $g_{\underline{\alpha}^{\prime} \beta^{\prime}}$ ). Then considering arbitrary frame transforms $e^{\underline{\alpha^{\prime}}}$ we can compute $g_{\underline{\alpha}} \underline{\beta}=e^{\underline{\alpha^{\prime}}} e^{\underline{\beta}_{\beta}^{\prime}} g_{\underline{\alpha}^{\prime}} \underline{\beta}^{\prime}$. Finally, we can redefine for a nonholonomic $2+2$, or $4+4$, splitting certain data $\left(\mathbf{g}_{\alpha \beta}, N_{i}^{a}\right)$, for which, in general, $\mathbf{C}_{\tau \alpha \beta \gamma} \neq 0$, and the corresponding to $\nabla, C_{\tau \alpha \beta \gamma} \neq 0$. Such construction with nonholonomic deformations are possible because vierbeins (11) may depend on some $N$-coefficients of a generic off-diagonal form of "primary" metric. The transformation laws of d-objects on nonholonomic manifolds with $\mathrm{N}$-connection are different from those on usual manifolds without $\mathrm{N}$-connection splitting (1).

Nonholonomic twistor spaces can be associated to any metric structure if a necessary type $h$ - $v$-splitting is defined by corresponding $\mathrm{N}$-connections. One of the important tasks is to formulate such conditions when certain nonholonomic deformations can be used for encoding exact solutions of Einstein equations in nonholonomic twistor structures and, inversely, to formulate nonholonomic twistor transforms generating exact solutions in general relativity and modifications. We shall provide such constructions in our further works.

\section{Acknowledgements}

The work is partially supported by the Program IDEI, PN-II-ID-PCE-20113-0256 and by an associated visiting research position at CERN.

\section{References}

[1] R. Penrose and W. Rindler, Spinors and Space-Time, vols. 1 \& 2. (Cambridge University Press, 1984 \& 1986).

[2] Yu. I. Manin, Gauge Field Theory and Complex Geometry. (Springer-Verlag, 1988).

[3] R. S. Ward and R. O. Wells, Jr, Twistor Geometry and Field Theory. (Cambridge University Press, 1990).

[4] S. A. Huggett and K. P. Tod, An Introduction to Twistor Theory. London Mathematical Society Student Texts 4, second edition (Cambridge University Press, 1994).

[5] S. Vacaru, Decoupling of Field Equations in Einstein and Modified Gravity. J. Phys.: Conf. Ser. 543 (2013) 012021; arXiv: 1108.2022v3.

[6] S. Vacaru, Branes and quantization for an A-model complexification of Einstein gravity in almost Kaehler variables. Int. J. Geom. Meth. Mod. Phys. 6 (2009), 873-909. 
[7] S. Vacaru, Einstein gravity as a nonholonomic almost Kähler geometry, Lagrange-Finsler variables, and deformation quantization. J. Geom. Phys. 60 (2010), 1289-1305.

[8] S. Vacaru, Two-connection renormalization and nonholonomic gauge models of Einstein gravity. Int. J. Geom. Meth. Mod. Phys. 7 (2010), 713-744.

[9] H. Rund, The Differential Geometry of Finsler Spaces (Springer-Verlag, 1959).

[10] M. Matsumoto, Foundations of Finsler Geometry and Special Finsler Spaces. (Kaisisha: Shigaken, Japan, 1986).

[11] A. Bejancu, Finsler Geometry and Applications. (Ellis Horwood, Chichester, England, 1990).

[12] A. Bejancu and H. R. Farran, Geometry of Pseudo-Finsler Submanifolds. (Kluwer Academic Publishers, 2000).

[13] D. Bao, S. -S. Chern and Z. Shen, An Introduction to Riemann-Finsler Geoemtry. Graduate Text in Math., 200 (Springer-Verlag, 2000).

[14] Clifford and Riemann- Finsler Structures in Geometric Mechanics and Gravity, Selected Works, by S. Vacaru, P. Stavrinos, E. Gaburov and D. Gonţa. Differential Geometry - Dynamical Systems, Monograph 7 (Geometry Balkan Press, 2006), www.mathem.pub.ro/dgds/mono/va-t.pdf and gr-qc/0508023.

[15] P. Stavrinos, Weak gravitational field in Finsler-Randers space and Raychaudhuri equation. Gen. Rel. Grav. 44 (2012), 3029-3045.

[16] A. P. Kouretsis, M. Stathakopoulos and P. C. Stavrinos, Imperfect fluids, Lorentz violations and Finsler csomology. Phys. Rev. D 82 (2010), 064035.

[17] N. E. Mavromatos, V. A. Mitsou, S. Sarkar and A. Vergou, Implications of a stochastic microscopic Finsler cosmology. Eur. Phys. J. C72 (2012,) 1956.

[18] J. Skakala and M. Visser, Bi-metric pseudo-Finslerian spacetimes. J. Geom. Phys. 61 (2011), 1396-1400.

[19] S. Vacaru, Finsler and Lagrange geometries in Einstein and string gravity. Int. J. Geom. Meth. Mod. Phys. 5 (2008), 473-511.

[20] S. Vacaru, Spinor structures and nonlinear connections in vector bundles, generalized Lagrange and Finsler spaces. J. Math. Phys. 37 (1996), 508-523.

[21] S. Vacaru, Spinors and field interactions in higher order anisotropic spaces. JHEP, 09 (1998), 011, p. 1-49.

[22] S. Vacaru and P. Stavrinos, Spinors and Space-Time Anisotropy. (Athens University Press, Athens, Greece, 2002), 301 pages, gr-qc/0112028.

[23] E. Cartan, Les Espaces de Finsler. (Paris, Herman, 1935).

[24] S. Vacaru, The entropy of Lagrange-Finsler spaces and Ricci flows. Rep. Math. Phys. 63 (2009), 95-110.

[25] S. Vacaru, Superstrings in higher order extensions of Finsler superspaces. Nucl. Phys. B 434 (1997), 590 -656.

[26] S. Vacaru, Spectral functionals, nonholonomic Dirac operators, and noncommutative Ricci flows. J. Math. Phys. 50 (2009), 073503.

[27] S. Vacaru, Finsler branes and quantum gravity phenomenology with Lorentz symmetry violations. Class. Quant. Grav. 28 (2011), 215991.

[28] S. Vacaru, Critical remarks on Finsler modifications of gravity and cosmology by Zhe Chang and Xin Li. Phys. Lett. B 690 (2010,) 224-228. 
[29] S. I. Vacaru, Minisuperspace twistor quantum cosmology. Studia Universitatis Babes-Bolyai, Cluj-Napoca, Romania, XXXIV, 2 (1990), 36-43.

[30] S. Vacaru and S. Ostaf, Twistors and nearly autoparallel maps. Rep. Math. Phys. 37 (1996,) 309-324.

[31] S. Vacaru, Application of Nearly Authoparallel Maps and Twistor-Gauge Methods in Gravity and Condensed States. (Department of Physics, University Alexandru Ioan Cuza, Iaşi, Romania, 1994) [in Romanian].

[32] G. Vrănceanu, Sur les espaces non holonomes. C. R. Acad. Paris 103 (1926), $852-854$.

[33] G. Vrănceanu, Sur quelques point de la théories des espaces non holonomes. Bull. Fac. Şt. Cernăuţi 5 (1931) 177-205.

[34] G. Vrănceanu, Leçons de Geometrie Differentielle. Vol. II (Edition de l'Academie de la Republique Poopulaire de Roumanie, 1957).

[35] A. Bejancu and H. R. Farran, Foliations and Geometric Structures. (Springer, 2003).

[36] J. Kern, Lagrange Geometry, Archiv der Mathematik. (Basel) 25 (1974), 438443.

[37] H. Akbar-Zadeh, Generalized Einstein manifolds. J. Geom. Phys. 17 (1995), $342-380$.

[38] C. Ehresmann, Les conexiones infinitèsmales dans un espace fibré différentiable. Coloque de Topologie, Bruxelles (1955), 29-55.

[39] A. Kawaguchi, On the theory of non-linear connections. I, II, Tensor, N. S. 2 (1952), 123-142; 6 (1956), 165-199.

[40] S. Casey, M. Dunajski and P. Tod, Twistor geometry of a pair of second order ODEs. arXiv: 1203.4158.

[41] S. Vacaru, Principles of Einstein-Finsler Gravity and Perspectives in Modern Cosmology. Int. J. Mod. Phys. D 21 (2012), 1250072.

[42] L. Berwald, On Cartan and Finsler geometries, III. Two dimensional Finsler spaces with rectilinear extrema, Ann. Math. 42 (1941), 84-122.

[43] S. Chern, Local equivalence and Euclidean connections in Finsler spaces. Sci. Rep. Nat. Tsing Hua Univ. Ser. A 5 (1948) 95-121; or Selected Papers, vol. II, 1994 (Springer, 1989.)

Sergiu I. Vacaru

Associated visiting research affiliation:

Theory Division, CERN, 1211 Geneva 23

Switzerland

and

Rector's Office, Alexandru Ioan Cuza University

Alexandru Lapuşneanu Street 14, UAIC - Corpus R, office 323; Iaşi, 700057

Romania

e-mail: sergiu.vacaru@uaic.ro

http://www .uaic.ro/uaic/bin/view/Research/AdvancedTheoretical

Received: June 21, 2014.

Accepted: August 03, 2014. 